\title{
Zur Ko-Konstruktion
}

\section{einer amüsanten Unterbrechung während einer argumentativen Auseinandersetzung}

NIKOLINA PUSTICKKI/RI:INHOLD SCHMITI

\section{EINLEITUNG}

Wir beschäfigen uns in unserem Beitrag mit einer Situation, die im Rahmen der beruflichen Ausbildung von Mechatronikern dokumentiert wurde'. Vier Auszubildende sind mit cinem Ausbilder in einer argumentatıven Auscinandersetzung engagiert. Ein weiterer Ausbilder kommt <ur Gruppe hinzu und zeigl sich sehr interessiert an dem Aufnahmegerät und den Kuphö̈rern. die aul dem Aklenschrank liegen, an dem dic Gruppe stchı. Da er nicht merkı. dass dic Siluation auch mit einer Vidcokamera aulge 2 eichnet wird, unterbrechen die Aus<ubildenden und ihr Ausbilder suk<essiv die Argumentation und machen sich über ihn lustig.

Eröffinet wird unser Beitrag durch eine theoretische Einleitung. in der wir aus multimodaler Perspeklıve dic Relevan von Ko-Konstrukiın als Erkennınisperspeklive aul' Interaktion charaktcrisiceren und dic Kon 2 epte eınführen. dic für unsere Analyse wichtig sind. Im analytischen Teil rekunstruieren wir im Detall den Prozess der schriltweisen Herstellung der unterhaltsamen Unterbrechung. Wir fragen dabei nach dem Beitrag der Beteiligten \&u deren Etablierung und reflektieren abschließend dic Funktionalität der Ko-Konstruktion für die argumentatıve Auscinandersetzung. Zum Abschluss des Beitrags stellen wir dar, welchen Nut<en cine multimodale Kon tion von Interaktion durch die analyscleitende fokussicrung aul $\mathrm{Ku}$-Konstruktion erhalten kann. und welchen Gicwinn für das Konzept Ko-Kunstruktion mı der Einnahme einer multimodalen Perspektive verbunden ist

I Die Autnahmen sınd im Rahmen des Disscrationsprojekts von Nikolına Pustickı entstan. den. Sic untersucht aus eıner multımodalen Perspektıve das Kommunikatıonsverhalten dicser Gruppe von Auszubildenden im llınblick auf ausbildungstelevante und ausbildungsie. Alexive Aspekte ihrer Selbstdarstellung. Diese Aspekte werden als "prototypische Präsenz. figurena konzeptualisier und erlauben nicht nur den Vergleich der Auszubildenden, sun. dem erlauben Ruckschlusse, wie die Auszubildenden ihr interakives Verhalten im Sinne eıner "Selbstprofiessionalısierunga den Ausbildern anbieten. 


\section{Theoretische Und konzeptuelle Rahmung}

Wir betrachicn das Konzept der "Ko-Konstruklion " nıcht so schr als crgebnisbezogenes Konstrukt, das auf von mehreren Betciligten arbeitsteilig gefertigte Produkte venweist. sondern als Erkenntnisperspektıve In diesem Verständnıs opericr es auf der - aus ethnomethodologischer Sicht formulıcrien - grundsätzlichen Aushandlungsqualıtät von Interaktion. dic unhıntergchbar als gemeınsame Hervorbrıngung zu verstchen ist. Das Kon 2 ppt "Ko-Konstruktıon « muss sich also - will es mıt Berecht1gung analyseleıtend und erkenntnisgenericrend sein - zu dicser grundsätzlichen Basis gemeinsamer Hervorbringung von Interaktion kontrastiv und spe

Worin nun liegı das Speatische und Kontrastıve, das dem Konzept inhärent Ist? Dic Beantwortung dieser frage ist gleichermaßen cinfach und schwicrig: Beı KoKonstruktionen muss es sich um eine besondere Form der gemeınsamen Herstellung von Intcraktion handeln. Oder crkenntnisperspektıvisch formulicrt: Dic Vorstellung von Ko-Konstruktion crölinet die Suche nach und die cmpirische Analyse von formen interaktiver Herstellung. beı denen die (iemeınsamkeıl der Orientıerung. die (ileichsinnigkeıt der Sinnproduktion und dic kollektıve Relevantsctzung thematischer oder pragmatıscher Aspekıc bestımmende Merkmale sind

Wir schließen uns damil der nachstehenden Delinilion der Herausgeber ihrem Beitrag an: "Wir delinicren daher , Kokonstruklion, als das gemeinsame Handeln von Interaklionspartnern eur Fortselzung ciner Interaklion auf ein Zicl hın. (Seite 24)

Unter einer ko-konstruktiven Perspektive haben wir es also nicht mit Interaktionskonstitution an sich - im Sinne cincr kollcktiv betricbenen machinery - zu tun. Viclmehr werden Mechanismen der Interaklionskonstllution dazu genutal, um gemeinsam cinc spezifische form sozlaler Bedculung zu schaffen. dic durch cin kolleklives Projekı getragen wird. Die empirische Eviden 2 cines solchen Projekıes ist naturgemäß schr unterschicdlich: Einc von $\angle w e i$ verschicdenen Beteiligten in einem sequenzicllen formulıcrungsprozess gemeinsam produzıcric Äußcrung "' gehör hicr chenso in den ko-konstruktiven Relevan 2 rahmen wic der "sprachlose" (jang als Duell aweicr Konlirmandinnen in Jer Eröffnungsphasc cines (jollesdiensies (Schmill $2012 \mathrm{a})$.

Beides sınd als Ko-Konstruktıonen $<u$ versichen, für dic charakıerıstisch ist, dass cın Betciligter zwar auch alleine eınc Äußcrung produzicren und den (iang <ur Ker $\angle C$ und zurück in dic Kırchenbank realısieren kann. damı jedoch eine gänzlıch andere sozialc Qualitat realisicren würde: chen keıne Ko-Konstruktion. dic dic betciliglen "Konstrukteure" auf speallische Weise sozial mitcinander verbindet. Wie der maxımale Kontrast zuischen der gemeınsam produzierien Äußerung und dem "gemeınsamen Gehen « schon andeutel. Ist die cmpirısche Eviden beı verbalen Ko-Konstruklionen offensichilicher als beı solchen. die im Medium des körperlichen Ausdrucks produzier werden und deswegen nur visuell wahmehmbar sind

2 Zu formen ko-konstruktiver Äußerungsproduktion siche beispielsueise Lemer 12002. 2004). Sacks (1992). DiazjAntakı'Collins (1996), Goodwın (198.3), Goodw'in'Goodw'ın (1986). sıchc auch Schmıtt (1998) zu "Unterstützenu als komplexem. gesprächsrhctorıschem lierfahren. 
Aus ciner multimodalen Sicht aul' Interaktion ist dic Vorstellung von Ko-Konstruktion gerade deswegen besonders interessant und inspiricrend. well ste das Augenmerk auf Siluationen und deren spezlfische Kunstilutionslogik lenkl. die man ohne diesen spealfischen Erkenntnıshinwels leicht in ihrer spezifischen sozlalen Qualitä der Sinnproduktion überschen uürde. Einc Herauslorderung liegl aus multimodaler Siche darın, das ursprünglich verbal delimere Kon 2 ept bei der Analyse anderer Ausdrucksressourcen be ¿ïglich scincr mulumodalen Tauglichkell <u reflekticren

Umgekchrt trägt dic mulumodale Perspektive für das Kon\&cpt der Ko-Konstruktoun dacu beı, bislang micht gehörte - weil nur sichtbare - Verhaltensweisen hinsichtlich ihrer ko-konstrukliven Qualitäl und Relevan sichtbar <u machen. Dies gilt beispeclsweise für "verbale Abstuncne als interaktive Belciligungswcisce (Heidmann Föh 2007; ssche auch die nachlolgende Analyse).

Ko-Konstruktion als ursprünglich verbales Konzept gewinnt letallich durch Konzepte, die im Kahmen einer multumodalen Konzeption von Interaktion entwickelt worden sind, an Varlanzreicheum und empirischer Sichtbarkelt. Eines der eentralen Kon<epte mit hoher Implikalivität für die ko-konstrukliwe Perspektıve ist mIntcrakIIonsensemble“" (Deppermann Schmult 2007. Schmill 2012b. 2013). Bcum "Inleraktionsensemblë" verlicen die Katcgorien "Sprechere und "llörere ihre bisherige Ze-

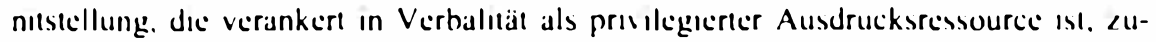
gunsten aller an der Interaktion Beteiligten. Dies geschich gän<lich ungeachtec der Frage, ob sie sich verbal oder in anderen Ausdruckstormen an der Interaktion beteilıgen. Erst bei cuner Kun<ciption. de vom gesamten tinscmble als <entraler Handlungsund Analysecinhelt ausgeht. kommen de Interaktionsbeiträge cerbal Abstinemter auch unter concr ko-konsiruklıven l:rkenntmısperspekuve in den Blick.

Interaklionsensomble als aus mulumodaler Sicht primärer Bezugspunkt jeglicher analytischer Anstrengungen. gecht eng einher mit dem Kon\&cept "Interaktionsraume (slche vor allem Mondada 2007. Schmult Deppermann 2007 und Müller Bohle 2007). Interaktionsraum ist eme im multumodalen Erkenntnis<usammenhang in a ischen etablicrle Vorstellung. de körperlich-räumliche Konstellationen als Grundlage und Fundament lür Interaktion lokussicrt. Die Betciligten stellen in der Interaktion und für spealische Zwecke ihrer Interaktion solche Interaklionsräume her. wobet sie sich der kunkretion räumlichen Gegebenhelten ihrer Umgebung als interakliver Ressource bedienen (Hausendori/MondadivSchmıll 2012).

Interaktionsensembles bilden in der Regel automalisch Interaktionsraume aus. mil denen Implıkationen für die Innen- und Außenstruklur (Schmult 2012b) verbunden sind. Sie können aufigrund ihrer körperlich-räumlichen Kunstellation von Außenstehenden problemlos wahrgenommen werden. In der Regel werden ste als vergesellschafiete Strukturen behandelt. für deren Störng oder Erwellorung spezllische Bedingungen vorliegen müssen. In vielen Fällen gilt ein Nicht-Respeklieren etablicrter Interaktionsriame als so<lal auliallig. Diese beiden in spe

3 Mondada (2007) hat exemplarisch und in prototypischer Hesc de llerstellung von Interatkllonsraumen (am Belspicl von Wegauskünften) als notwendige Vorausictzung der interakliven /.weckrealisterung systemattisch verdeutlicht. Sie bictet cine detalllerte Darstellung. in welchem Virhaltnis . Intcraktionsraum zu Vorstellungen uic "Situatione oder "Kontcxte stcht. 
be zogenen Konzeple (Schmılt 2013) spiclen für unscre nachfolgende Analyse cinc wichuge Rolle.

\section{FALLANALYSE}

Im rolgenden wollen wir rekonstruicren, wic die amüsante Unterbrechung eıner argumentatıven Auscinandersetzung schrillweise hergestellt wird. Wir werden uns dabeı auf die unterschicdlichen Betträge der Beteiligten konzentrieren, um zu schen, wer wann welchen Beitrag <ur Unterbrechung Ieıstet.

\subsection{Die argumentative Auseinandersetzung}

Dic argumentalive Auscinandersetzung wird verbal von dreı Betciligten getragen: von $\angle$ wel Aus $\angle u b i l d e n d e n$ (Maxi und Hannes) und ihrem Ausbilder (Weber). Zwei weitere Aus $<u b i l d e n d e$ (Chris und Peter) sind verbal abstınent. Trot<dem sind diese als Mitglieder der Ausbildungsgruppe und somut des Interaklionsensembles in der Auscinanderset<ung nıchı nur präsent, sondern aktıv an deren Konstltutıon betciligt. Aulgerund ihrer Präsen L. Ihrer körperlıchen und blicklichen Orıentıcrung sou ie ihres Aufmerksamkeıts-Dısplays verkörpern sıe die zweı Hälfte der Gruppe. Somı werden die thematisch-pragmatıschen Relevanzen, dic verbal nur durch Maxi und Hannes bearbeıte wurden, als Relevanz der gesamten Ausbildungsgruppe verdeutlıcht [Bıld 1].

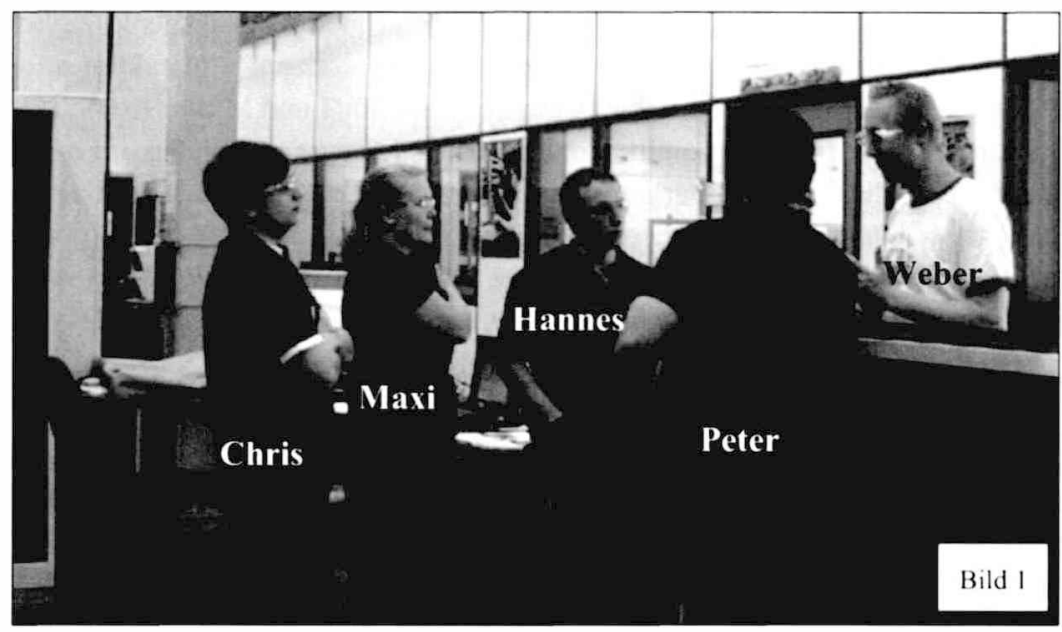

Dıc Aus<ubıldenden sind mit der schrıftlichen Ausarbeıtung eınes halbjährıgen. prüfungsiclevanten Projekies in. Wenn sie die Dokumentation mit der Post an die Prüfer schicken. Wird sie wahrscheinlich nicht mehr rechizeilig ankommen. Daher lordern

4 Aufgrund der komplexen (jleichzeıtıgkeıt der Interaktıonskonstıtution verzichten wir dabeı auf eıne konsequent sequenzanalveısche Rekonstrukıon des (ieschehens. 
sie von ihrem Ausbilder cinen Dienstwagen, um die Dokumentation sclbsi am Bcslimmungson abzugeben. Dieses Ansinnen wird verweigern. Dic Aus<ubildenden formulieren daraulhin eine indirckle Schuldzuweisung an den Ausbilder: Sie seien primär in Verzug geralen. weil er lür sie in den letzlen Tagen zu wenig Zeil hatte. Diesc Schuldzuweisung weisi Weber zuruck. Er formuliert seincrsells, dass sich dic Gruppe organisatorisch und arbellstcilig vertan hat und deswegen nicht rechizelleg ferlig geworden ist.

81 Weber

82 Hannes

Während Weber seinen (iegenvorwurf formuliert (ihr wussle' dass dee lermin STEHT), erscheint im hinteren Teil der Ausbildungswerkstall Offenheim, cin weitcrer Ausbilder. Er bewegl sich auf einem zentralen Durchgang der Ausbildungswerkslall, der hınter dem Akıenschrank vorbeılühn, an dem sich der ersic Ausbilder aulhält. Dic Projektıon scines Laulwegs führt thn entweder in cines der von ihm aus gesehen linken Besprechungs 2 Immmer oder an der diskulıerenden Gruppe vorbei.

\subsection{Annäherung und Etablierung des zweiten Ausbilders (während der Argumentation)}

Während sich Oflienheim der diskutierenden Gruppe nähert, crfolgt eine erste ReakIıon auf scine Präsenz. Weber. der gerade damil beschäftigt ist, die Schuldzuweisung aus zuhebeln. wendel während seiner Ausführungen kur seinen Kopl in Richtung des herankommenden Kollegen. Kur $<$ daraul dreht sich auch Maxı mil ihrem Oberkörper und Kopi nach lınks in Richeung des Herannahenden [Bild 2]".

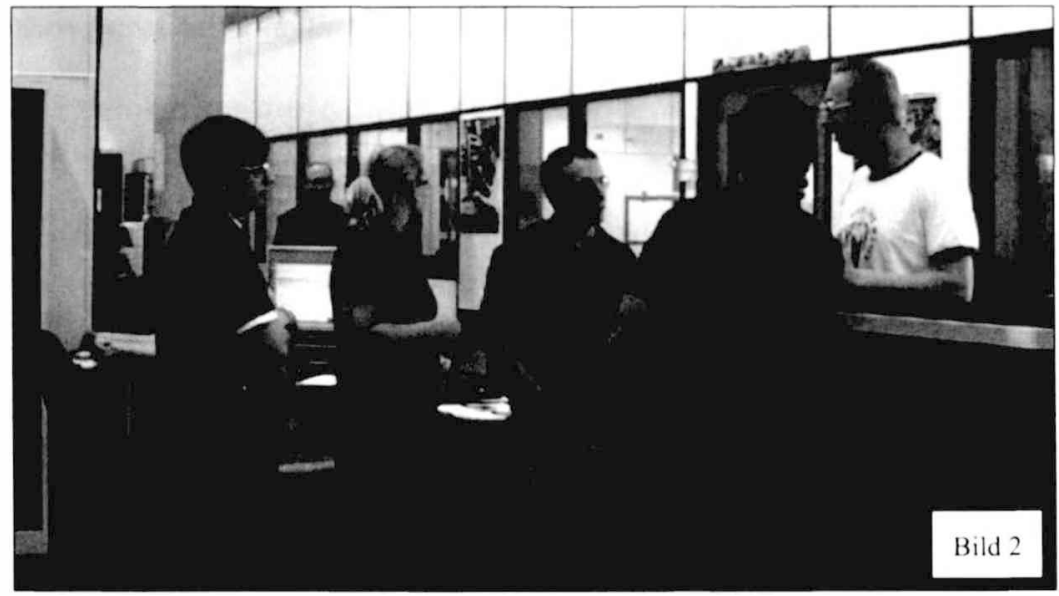

90 Weber ihr $\underline{\mathbf{k r}}$ iegt geNUG hilfe wenn irgerdwas ist,

5 Fillmarkierung und Unkerstreschung virueisen auf dic Zuordnung von Bild und Transkript. 
Es ist sehr wahrscheınlich. dass auch Peler aufgrund scincr Posılton und Körperausrıchtung den Ausbılder wahmımmt. Dicser läuli gewisscrmaßen dırekı in den Wahrnehmungsbereich des Auszubildenden

Während Offenhein scincn Weg fortsctzl, deutel seinc Koplposition auf scinc Orıentıcrung an der Ausbildungsgruppe. Scın Blıck ıst aut dic Ablagefläche des Aktenschranks gerichtet. aul der sich das rot leuchtende Audioaulnahmegerät befindet. das dort zur Dokumentation deponiert wurde

Chrıs ist das nächste Mitglıed des Interaktıonsensembles, das den herankommenden Ausbilder wahrnımm! [Bild 3].

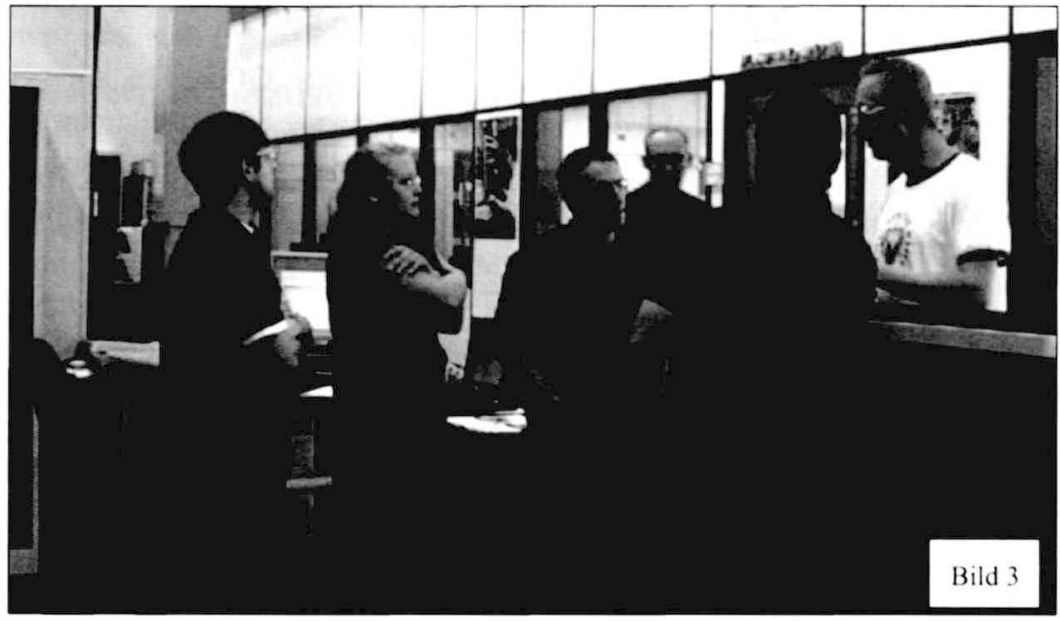

93 Maber wISST wie chs mein

Anders als beı Weber und Maxı. bleıbt Chrıs ${ }^{\circ}$ Blıck länger auf Offenhem gerıchtet. während die Argumentation weitergeht

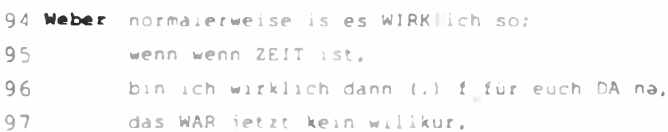

Mit seınem Blıck folgt Chris Offenheın. der in Zwischen in unmıltelbarer Nähe der Ausbildungsgruppe angelangt ist. Das körperlıche Verhalten des herankommenden Ausbılders und vor allem scın kontinuierlıcher Blıck aul das Audioaufnahmegerät. verdeutlichen eine thematısche Relevanz, die seinen weiteren Giang mitstrukturiert: Er dreht seinen Oberkörper entgegen sciner Laufrichlung nach rechts in Richtung der Gruppe eın.

Seıne gesamte Präsenz, vor allem jedoch dıc blıcklıche Fixıcrung des Aufnahmegeräts sınd Ausdruck einer exploratıven Orıentıcrung. die autgerund threr Dauer und Expressivität sou ic der unmiltclbaren Nähe zur diskutıcrenden Ausbıldungsgruppe. das Potenzial besilzt. Wahrgenommen zu werden. Das Interesse Offenheims und dic Ausschlıcßlıchkeıl seıner (Orıentıcrung auf das Aufnahmegerät drängen sich geu issemaßen der Wahrnchmung auf:

Chris. der das Verhalien Offenheims seil cinıgen Sekunden blicklich verfolgt hat. kommentıer den exploratwen Blıck zunächst mut eınem (irinsen. Dieses ereignet 
sich, als der Ausbilder seinen Laulweg unterbricht und sich gegenüber von Peter körperlıch nach vorne aul den Akınschrank schiebi. Don verankert er sich (Müller Buhle 2007) und begınne für mehrere Sekunden regungslos aut das Aufnahmegeräı «u starren (Zeılen 94-97) [Bıld 4].

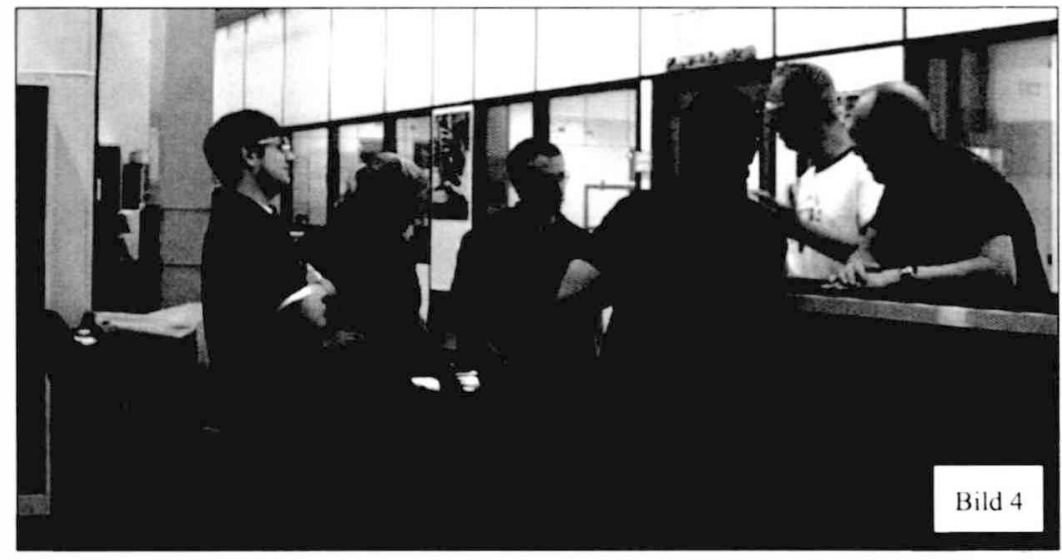

99 Weber ${ }^{\circ}$ isch renn jetz WEG,

So clabliert sich der Hinzugekommene aul markante Weise im bereils existlerenden Inleraklionsraum der Ciruppe Scine Integration geschicht ausschlicßlich körperlich. er ist verbal abstınent und interessien sich offensichllich michi für die themalischpragmalischen Relevan 20 der (iruppe. ter incegriert sich $\angle$ war in den bercils exislicrenden Interaktionsratum, wird jedoch niche tinsemblemuglicd Scine Orienticrung liegl ausischließlıch aul der visucllen Exploration der Siluation. die er rasch als "Audioaulnahmesiluation ce erkennt

\subsection{Exploration der Aufnahmesituation durch den zweiten Ausbilder}

Während der Exploralion oriention sich Olilenhem an den aul dem Aktenschrank liegenden Gegenständen (das Aulnahmegerät sowı de Kupthörer). Diese Gegenslände werden zu signifikinten Objekten, die sein welteres Verhalten weltgehend strukturieren. Die Exploration der Siluation nimml cine gewisse Zell in Anspruch, in der er sich mil der individuell autierlegten themalischen Relevan ohne ()rientierung aul de anderien Beteilıgien beschäligt. Seın exploratives Verhalten stellt jedoch eın Angebot für dicjenigen Enscmblemitglieder dar. dic es aufigrund ihrer arbeitsteiligen Einbindung in die argumentative Auscinandersetzung wahmehmen und vertolgen können. in erster Linic also ('hris und Peter, die verbal abstinenten Teamventeter. Es

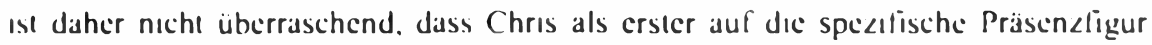
(Schmıll 1992a. b) des \&weiten Ausbilders reaglert. Er kummentierl das Verhalien des zweilen Ausbilders, das er zunächst mit cinem ersien (irınsen bedache hat. nun auch mu Lachen (Zeile 101). 
Chris indizicrt damit dic thematısche Relevanz und Altraktion, dic durch den hinzugekommenen Ausbilder in den Interaktıonsraum des Ensembles importicrt wurde. Ab der Verankerung an der Werkbank und dem über mehrere Sekunden andauemden regungslosen Starren auf das Aufnahmegerät hat sich Offenheim auch in Strukturen crhöhter Wahmehmungswahmehmung (Hauscndorf 2003) "verankerte. Ab dicsem Zcitpunkt ist es für ihn kaum noch möglich, dic Situation ohne Konsequenzen (beispiclsweise cinem Kommentar) ¿u verlassen, da er sich mit sciner exploratıven Präsenz als zwar verbal abstinenter. aber relevanter Teilnehmer im Interaktionsraum pusitionicrt hat. Spätestens als er sich dic Kophörer aufsetzl, realisien er ein offensıves Angebot zur Wahmehmung und cine potenzıclle Offerte für fokussicrte Interaklion

\subsection{Reaktionen der Ensemblemitglieder und Etablierung eines zweiten Fokus}

Das performative Potenzial der Präsenzfigur Offenhems ist gecignet, dic Orienticrung einzelner Ensemblemitglieder auf sich zu lenken und von der argumentativen Auscinandersetzung abzuziehen. Dies birgt in Ansätzen die Gefahr. dass durch die Reaktion auf scine Präscne cin zweiter, kontrastiver fokus im Interaktionsraum ctablıcrt wird. Um dıeses (icfährdungspotenzial des kürperlichen. gestıschen. mimischen und blicklichen Verhaltens für das Interaktionsensemble zu verstehen. wollen wir sein Verhalen nachfolgend im Zusammenhang beschreiben.

\section{Offenheim (2. Ausbilder)}

Nachdem Offenhem für mehrere Sckunden regungslos auf das Aufnahmegerät gesturrt hat. blickt er zu Chris, der scincrscits zu thm schaut. Im Moment ihres Blickkontakts gehı Chrıs' anfängliche (irınsen mit geschlossenen Lippen in eın leıses, aber breites Lachen mit geöffnetem Mund über, bei dem (hris scine Zühne «cigt.

Der Blıck Offenheims geht zunächst wieder zurück zum Aufnahmegerät und folgt dann dem Kabcl. das vom Aufnahmegerät <u den Koplhürem lührt. dic links neben ihm auf der Schrankoberfläche licgen [Bild 5]. 


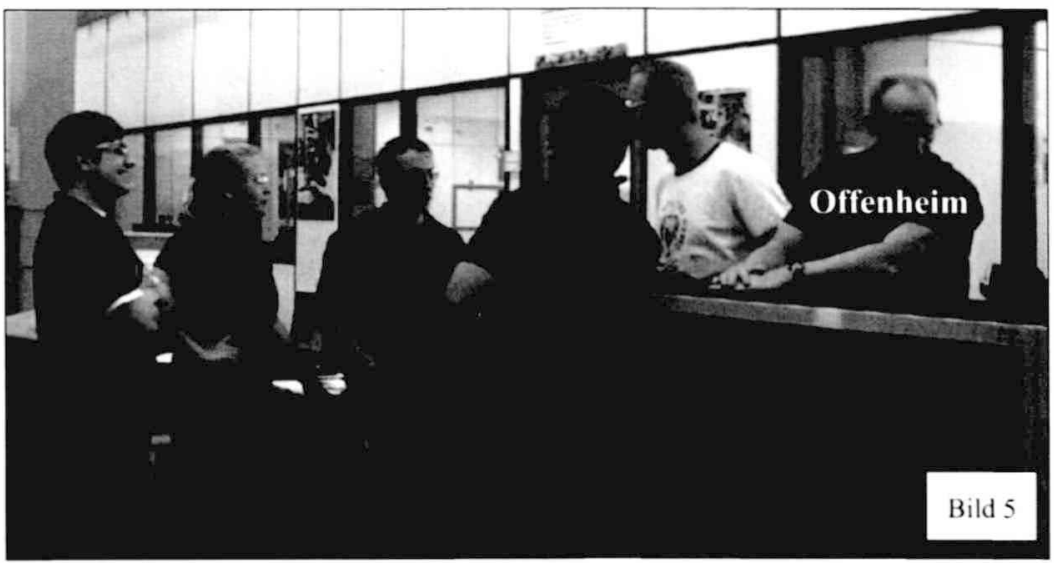

105 maxi hirk ch so dass w: r die letzcer. zwe ta:ge,

Nachdem er die Kopfhörer als <ugehörıgen Bestandicil des Aulnahmegeräts crkannı hal. kehri sein Blick wieder \&um Aulnahmegeräl zurück Nach einem kur\&en Moment dreht er sich schnell $<$ den Kuplhörern hin und greili bereits in der Drehung mil seiner lınken Hand nach diesen Als Olfienhem die Kopthörer berühri. gechen sein Blick und seine Kopforchung <uruck <ur Mille des Interaklionsraums. Dann set<l er sich die Kupthörer aul und siellt anschließend kun [Bild 6].

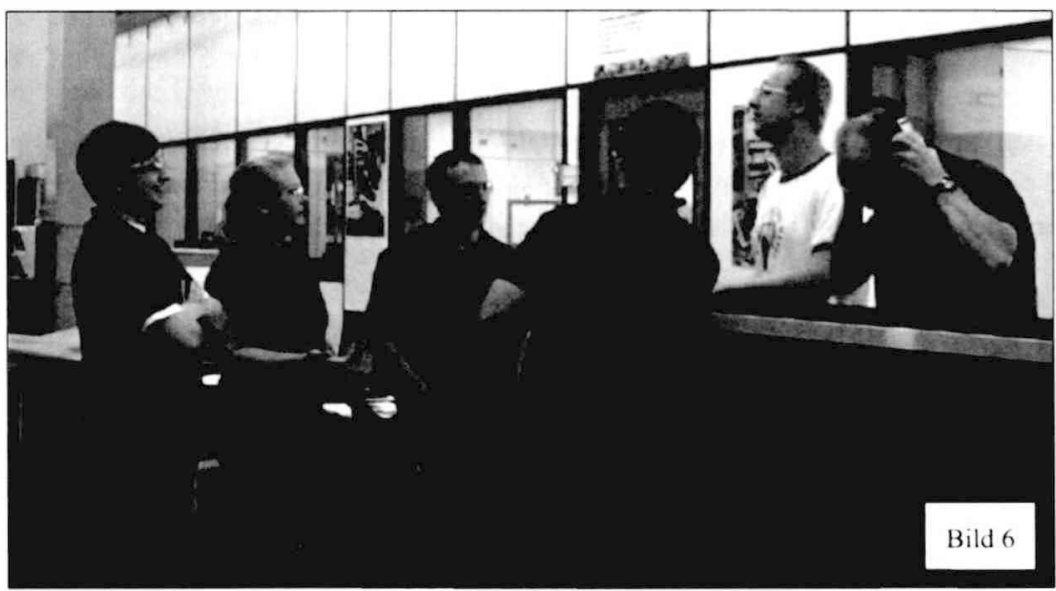

112 Maxi WFil $1.1<\langle a t i>$ sie nich: da waren und wir warten mussten und

Dabeı blickl er nach unten, als wüde er sich intensiv aul sein Handeln kun zentrieren

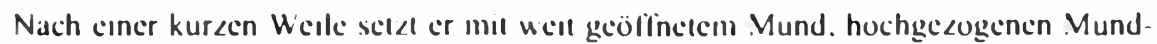
winkeln und ciner expressiven Bewegung die Koplhörer ab. Seın Blıck gechı <urück zum Millelpunkı des Interaklionsraums Nach Ablage der Koplhörer realisien er einen silualionsrellexiven Kommentar: Er hebt den Zeigetinger seiner rechien Hand in Richlung der Auszubildenden. ganz so. als wolle er sagen: waha. Achlung. aulgepassi!! [ [Bold 7]. 


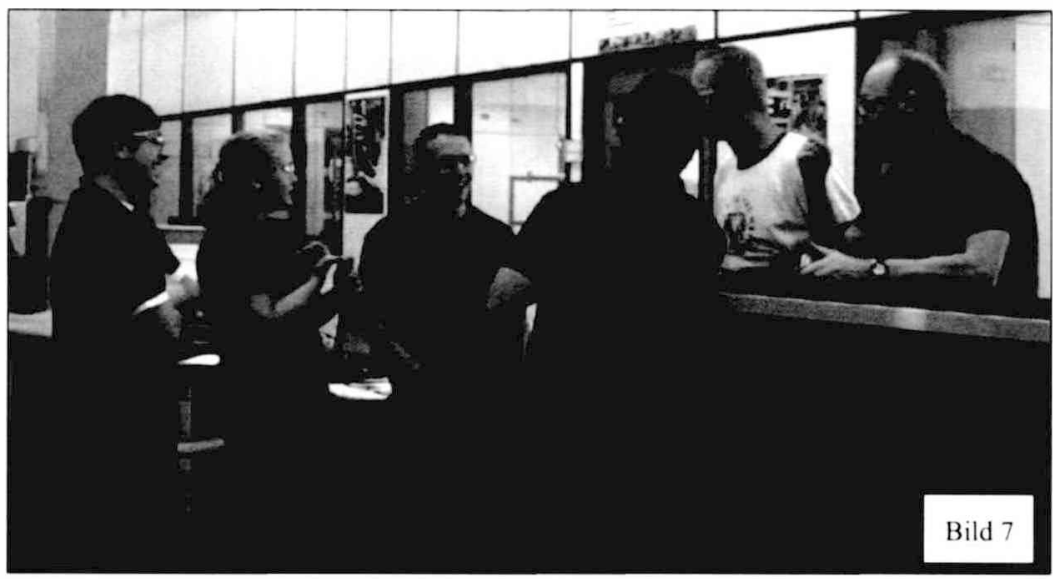

113 Maxi (.) weil wir konnten die die die PLÄne nicht vollenden.

Danach numme er witeder cine statısche Körperhaliung eın und fixien emeut regungslos das rol leuchtende Aufnahmegerät. Die ganze Zeıl über hal er seinen Mund geöfinct. Er bewegt seinen Blıck lediglich für einen kurzen Moment vom Aufnahmegerät $<u$ den Aus<ubildenden und verfällı dann für 6.5 Sckunden emeut in cine Bewegungsslarre.

Dann schaut Weber \&u ihm. Nachdem Offenherm bemerkt, dass scin Kollege ihn anschaut. dreht er scinen Kopf nach rechts und blickt ihm ins (jesicht, wobei scin Mund noch immer halb geöfinet ist. Scın mimischer Ausdruck deutet auf Folgendes; Er hat noch keıne stabile Hypothese darüber, um was es in der Situation eigentlich gehı.

Aul seinen reflexiven Kommentar mittels Heben des gestreckten Zeigelingers und sein expressives Starren reagien nun Hannes. Er produzicr die crste verbale Reaktion des Enscmbles auf dic Präsenz des zweıten Ausbilders.

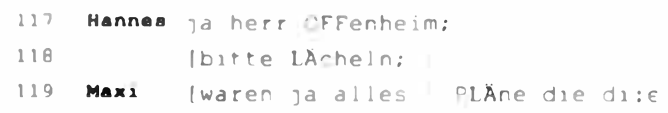

Mil der Äußerung ja herr OFFenheim: hille LAcheln: gibe Hannes zu verstehen, dass dic Siluation nicht nur audilıv, sondem auch visucll aufgęeichnet wird. Oflenheim hat also im Rahmen seiner bisherigen Exploration das wichtigste Detail überschen: Die Kamera und die Kameratrau, die etwas abseits der (iruppe stehen. Offenhems Blick gehe daraufhın sofort dorthın: Er starrt mit halb geöfinetem Mund und irritiertem Blick (scheinbar entgeisiert) in deren Richtung und verlisst schnell und mit erhobenen Händen den Schauplat< [Bild 8]. 


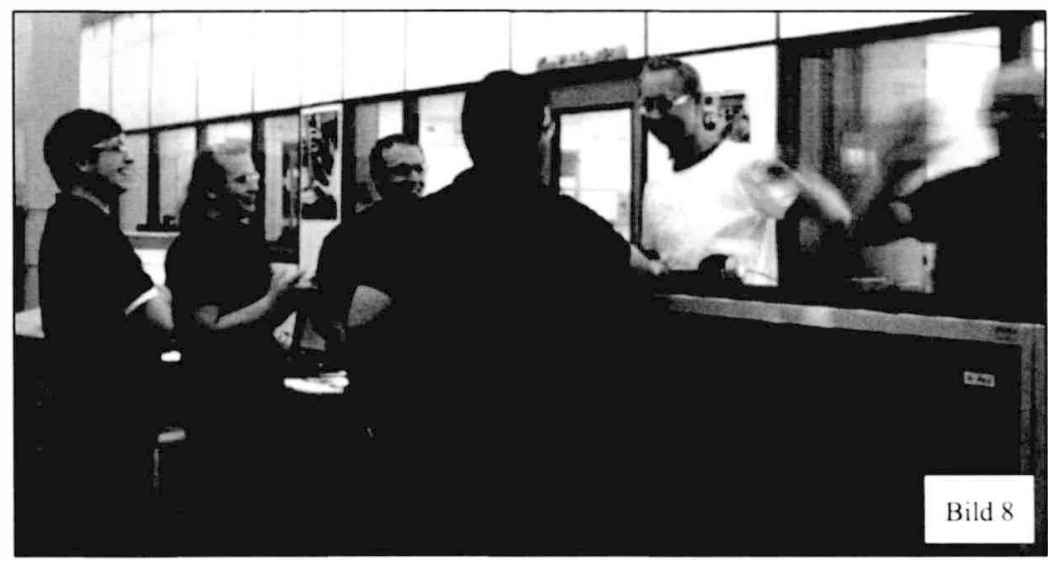

12 Poter ho hm bitce LAschelri:

Ollenheims Präsen . die in 2 wischen nichı nur von verschiedenen Belciligten wahrgenommen, sundern durch Hannes auch verbal thematisiert wird, führt \&u einer sukzessiven Neu-fokussierung des Ensembles. Als Maxi (als elabliene Sprecherin und Vertreterin der argumentatıven Auscinandersetzung) ihren Ietzlen Zuhörer (Weber) durch diese Neu-Fokussierung der anderen verlierl, gewinnt die Präsen $\angle$ des $\angle$ weiten Ausbilders den Stalus cincs zweilen, kunkurricrenden fokus. Wie dieser suk 2 essive rokuswechsel hergesielle wird und wie sich die ein 2 Inen Mitglieder des Ensembles an dessen Elablierung beleiligen, werden wir im Folgenden darsiellen.

Während der interaklionsraumlichen Integration des \&weiten Ausbilders belindel sich das Interaklionsensemble an cinem Punkt der Auscinandersel<ung, beı dem vor allem Maxı die Gruppenınteressen akııv vertrılt.

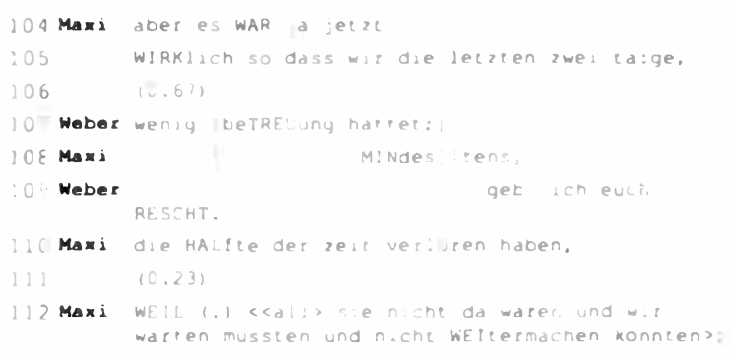

Maxı belont. dass ihre Ausbildungsgruppe aufgrund des Wartins aul Weber Zeil verloren hat und daher die geturderien Arbeiten nichi lertigstellen kunnte. Damil wird dem Ausbilder implizil eine Milschuld gegeben. Sic beginn dann dic Aulgaben aul<u<ählen. die deswegen nichı «eilgerechı bearbeiteı werden kunnien:

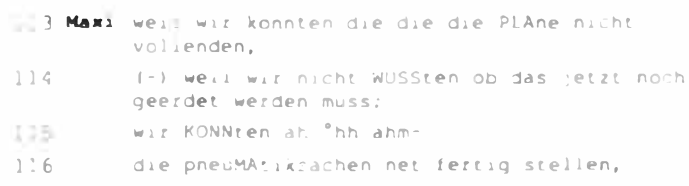




\section{Chris}

Während Weber. Maxi und Hannes die Argumentation bearbeiten, reagien Chris. der als verbal Abstınenter von der argumentatıven Vertretung der Gruppe gewissemaßen freıgestellt ıst. frühzeılıg und als erstes Enscmblemitglıcd auf die Angebotsqualıät der Präsenz von Offenheim. In Chris Mimik zeichnet sich zucrst ein Lächeln ab. gcfolgt von einem breiten Girınsen, beı dem seıne Zühne zum Vorscheın kommen, das schlicßlıch zu einem Ieısen Lachen wird (Zeıle 101 ). Vor dem Auftauchen des zweiicn Ausbılders war seıne körperlıche Präsenz durch eın kontinuicrliches Wippen von den fersen auf die Zchenspitzen und zurück charakterisıert. Chris hört mit dem Wippen auf. als Offenhem bereits in die Situationsexploration eingetreten ist und dieser zum ersien Mal seinen Blick auf die Mitte des Interaktıonsraums richtet. Nun beginnt sich Chris' Oberkörper rhythmisch unter Lachen zu bewegen. Als Offenhem erstmals das Aufnahmegerät starr fixiert. wirli Chris cinen ersten Blick in die Kamera [Bild 9].

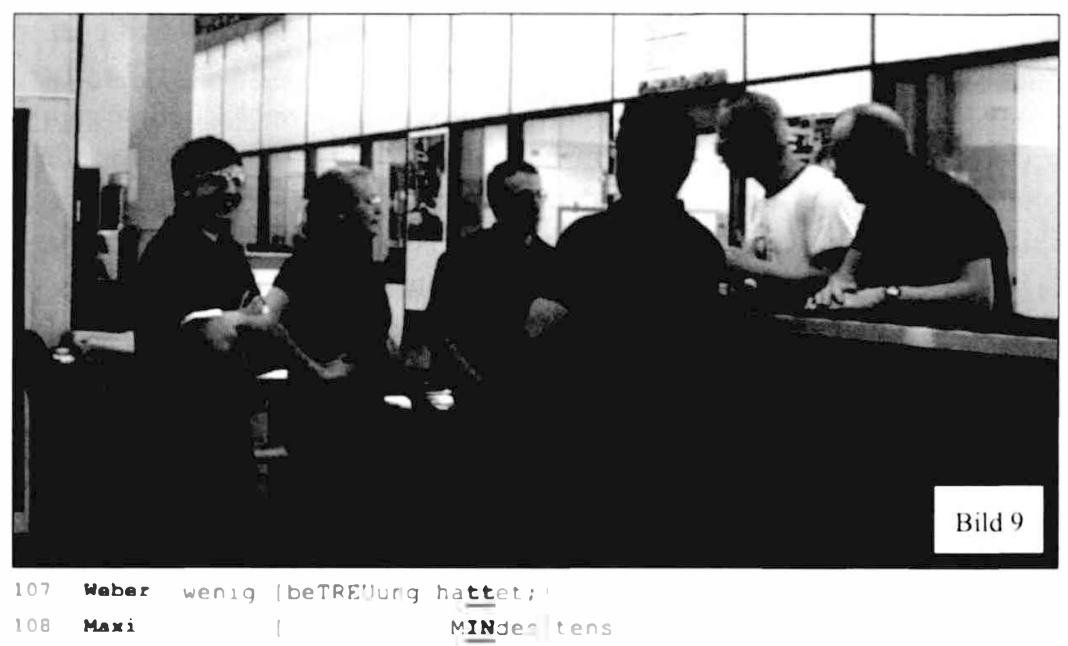

Chrıs ist darüber informicrt. dass dic Siluation nicht nur mithilfic von Audio-, sondern auch mit Vıdeoaufnahmegeräten dokumenticrt wird. Über dieses Wissen verfügt Offenheim nicht. Er weiß daher nicht, dass ihn dic Vidcokamera bereits seil seiner Elablicrung im Interaktionsraum und beı scinem Versuch. die Relevanz der Situation $\angle u$ rekonstruieren. dokumentien. Dem Lachen des Aušubildenden liegt also das Motiv der "versteckien Kamera" zugrunde. Dicses Motiv hesıl८l <um aklucllen Zeılpunkı ausschlicßlich individuclle Relevanz. ('hris wirft dann, noch immer mit cinem lautlosen Girınsen im Gesıcht, eınen Zweiten Blıck in dic Kamera, als Offenheim dic Kopfhörer aufsetkt.

Während des Aufset<ens der Kopthörer bestehen bereıts zwei sınguläre Orıentıcrungen von Chris und Hannes auf die Aulfälligkell Offenheims. Chris' laullose Lachen und Grinsen werden mil der Dauer der Exploration des Ausbilders zunchmend expressiver. Erst nachdem Peter auf das Hehen des Zeigefingers von Offenhem reagıert hat und beginnt, seıne Körperpositur zu verändert, gibl es den ersten Blıckkonlakı zwischen Peler und (hris. Mit diesem 13lickkontakl erfolgl die erste dyadische Relevantsctzung von Offienheims Präsenz [Bıld 10]. 


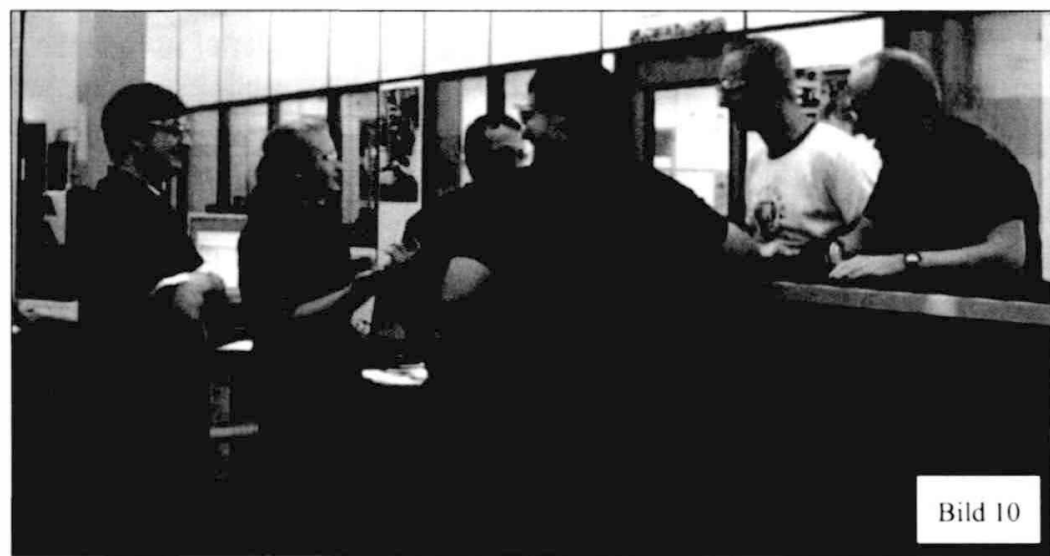

110 Maxi (-) well wir nichit wusster ob Jas jetzl roch ge ex jet werder

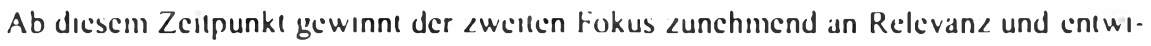
ckelt sich zu cinem laklıschen Kunkurrenzlokus für das Interaklionsensemble. Nachdem Peter den Blickkontakt mil Chris aufigelöst hat und seinen Blick wicder auf Offenheim rıchtel, blıckl auch Chris - mit zugeknıfienen Augen und zusammengepressien L.ippen - zu Ofienhem. Er drehe dann seinen Kupl «u Hannes, als dieser die crste Äulscrung an Ollionheim richtet.

\section{Hannes}

Gchen wir nun wieder an den Antang des Segmenis <urück und kon<entrieren uns auf die interaklive Prasen 2 von Hannes. Die Gruppe belindel sich noch in der verbaIen Aushandlung mı Weber, während Offienhem sich bereils an der Werkbank verankerl hal. In dieser Phase blickı Hannes, ohne seine Körperposilur \&u verändern. kur $<$ nach unten in seinen Nahbereich und monilon dann. nachdem er den Blick wicder angehuben hat. Weber und Oflenheim. Seın Blick folgt dann dem explurativen Verhalien des zweiten Ausbilders. Als Offenhem nach den Kopthörem greift und dicse aufsel<l, laucht in Hannes Gesicht ein Lächeln aul. das den Blick aut seine Zähne lreigibı [Bıld II].

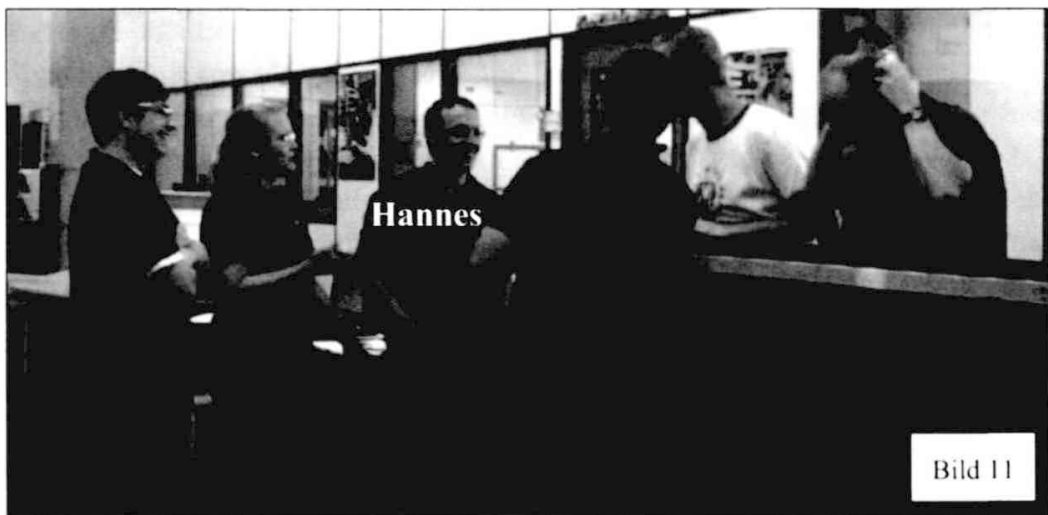


Mil den Händen in den Hosenlaschen und dem lautlosen Lachen auf den Lippen verfolgı Hannes die Exploration Offenheims. Seinen Blick wendel er nur cinmal kurL ab. um in die Kamera zu schauen. Als Offenhem begınnt, seıne Bewegung für 6.5 Sekunden einzufrieren, verlıcrı sıch Hannes Lächeln. und er fixıcrt das auf der Werkbank lıegende Audıaufnahmegerät. Zeilgleıch mit Weber. der seinen Blıck auf Offenhem richtel, blıckı Hannes kur zur Kamera und dann wieder zu Offenhem. Danach kommentıcr er dessen Verhalien mit der bercits zilıcrten Äußcrung ja herr Oflenheim: hille LAcheln: (Zcilen 1178.).

\section{Peter}

Peters Verhalien beı der Etablicrung des zweiten fokus ist schwer zu heuricilen. da cr als cınzıger mı dem Rücken zur Kamera sıchı. Pcter Isı an den Akıcnschrank gelechnt und körperlıch auf das Innere des clablicrten Interaktıonsraums ausgerıchict. Erst als Offenheim seinen Zeigelinger hebı und die Situation geslikulatorisch kummentierl, reagierı Peier für uns erkennbar aul die Präsenz des zweilen Ausbilders [Bild 12].

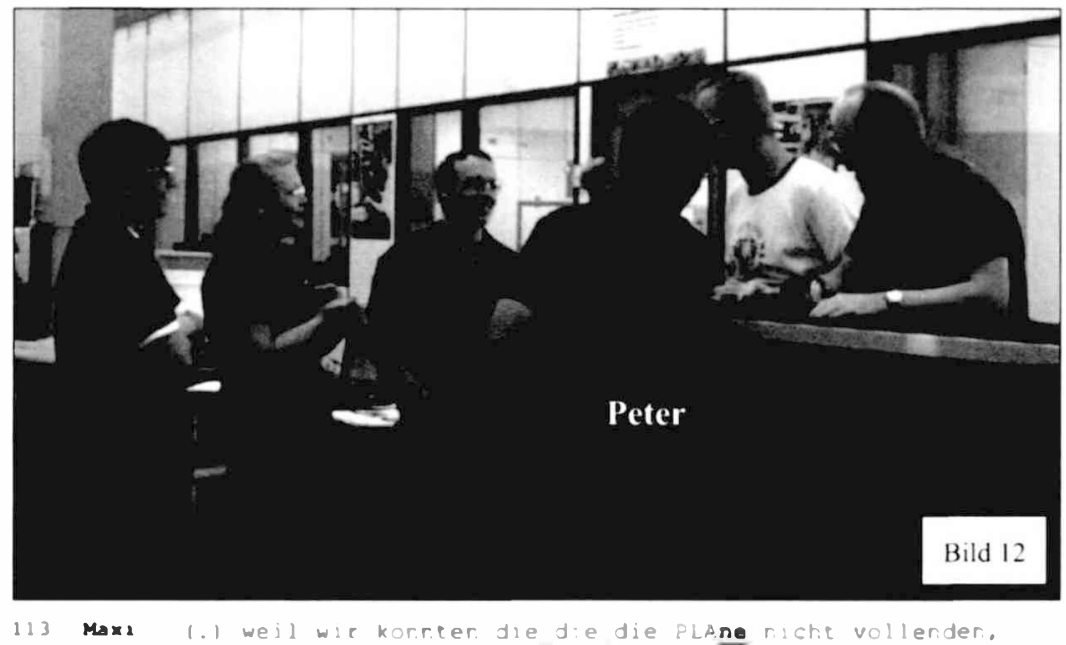

Peler wendel seinen Kopl nach rechts zum slarrenden und ..eingefrorenen" Offenheım. der ihm dirckı gegenüber am Schrank sıchı. Danach verschicbl Peler scine Position elwas nach lınks und blickı dabei \&u Chris. Beide schauen sich lachend an. uährend sich Peicr vor laullosem Lachen kure schültelt. Chris und Peter etablieren durch diese Aktıvität cıne Dyade. in der sie nichı mehr aul dic argumentalıve Auscınandersetzung orientien sınd. sondern ausschlıeßlıch dic Relevan Offenheims bearbeilen. Das Interaktıonsensemble hat sich also ausdifferen\&ier in eine Dreicrkonstellation. In der von Maxı. Hannes und Weber Fokus I weiler hearbeitel wird. und in cıne Dyade. die sıch mimısch und vokal mil Fokus 2 beschäliıgl.

Während sich Chris und Peler bereits für dic anderen Beiciligten wahrnehmbar auf den zweiten Interaklionsfokus orienlicren, Isı Hannes auch nicht mehr 100 Prozenı bei der Sache. Ledıglıch Maxı und Weber sınd nuch ausschlıcßlıch mi der Argumentation beschäftugt und als einıige Betciligle verbal aklıv. Das ursprünglıch fün föplige Ensemble ist nun also auf cınc Dyade geschrumpft. Dic dreı anderen 
Mitglieder sind mehr oder weniger deutlich der Altraktivitat und "Ablenkung" erlegen, die das explorative Verhalten Offienhems besitzt. Dic Mehrheit der Ensembleimitglieder hat also bereits einen fukuswechsel realisiert.

\section{Weber (1. Ausbilder)}

Die zunchmende Relevanz des \&weiten Interaktionslokus kupien sich auch in die argumentatıve Auseınandersetzung. Nachdem Weber mil wenig heTREUinng hallet: geh ich euch RESCHT (Zeilen 107ff.) auf Maxis Vorhaltungen reagien, beginnt auch er sich auf den zweiten fokus zu orienticren. Während er körperlich und blicklich weitcrhın aul Maxi ausgerichtet ist und nickend aul ihre Argumente reagien, set<t als erste Reaktıon auf Otfinheim in Webers Gesicht bereits ein erstes Lächeln ein.

Weber ist also cbenfalls in eine bi-fokale Orienticrung eingetreten, die sich mittels unterschiedlıcher Ressourcen ausdrückt. Während er körperlich weıterhın aul dic Bearbcilung des primären fokus ausgerichtet ist, reagien er mit einem Lächeln suwic einer leichten Unruhe im Oberkörperbereich auf die sich aufdrängende Relevanz seines Kollegen und die bereits von (hris. Peter und Hannes volleugene Umoricntierung. Dadurch verlagert sich dic Struklur der Enscmbleorienticrung wester. Zunächst existierte cine verbal aktive Triade (Weber. Maxi und Hannes), die mit der argumentatıven Auscinanderset<ung beschälitigt war. (jleicheciltig gab es einc Dyade (Chris und Peter). die bereits den \&weiten fukus ohne Verbalität bearbeitet. Nachdem Hannes sich nun ebenfalls auf den $\angle$ weiten fukus orientier, veränder sich die Aufimerksamkell des Ensembles <u einer neuen kullektıven und kunkurrierenden Orientierung. Auch Weber kann sich der nun immer stärker werdenden Relevan rukus nicht mehr gänzlich entzichen.

Nunınchr ist es Maxi, dic als einaige weiterhın ausschließlıch die Gruppenınteressen veriritt. Das explurative Verhalten des zweiten Aushilders und die immer offensichilicher werdenden Reaktionen von Chris. Peter und Hannes verstärken sich wechselseltig und führen zu eincr Hochstufung des zweiten fokus, der sich altch Weber aufdrängt.

Als Petcr den ersten Blıckkontakt mı (hris auflöst und sich körperlich aul den <weiten Ausbilder ausrichtet, schaut auch Weber auf das Aufnahmegerät und wendel dann scinen Kopf nach links zu seinem Kollegen, der in einer side-by-side-Pusition unmiltelbar neben thm steht [Bild 13]. 


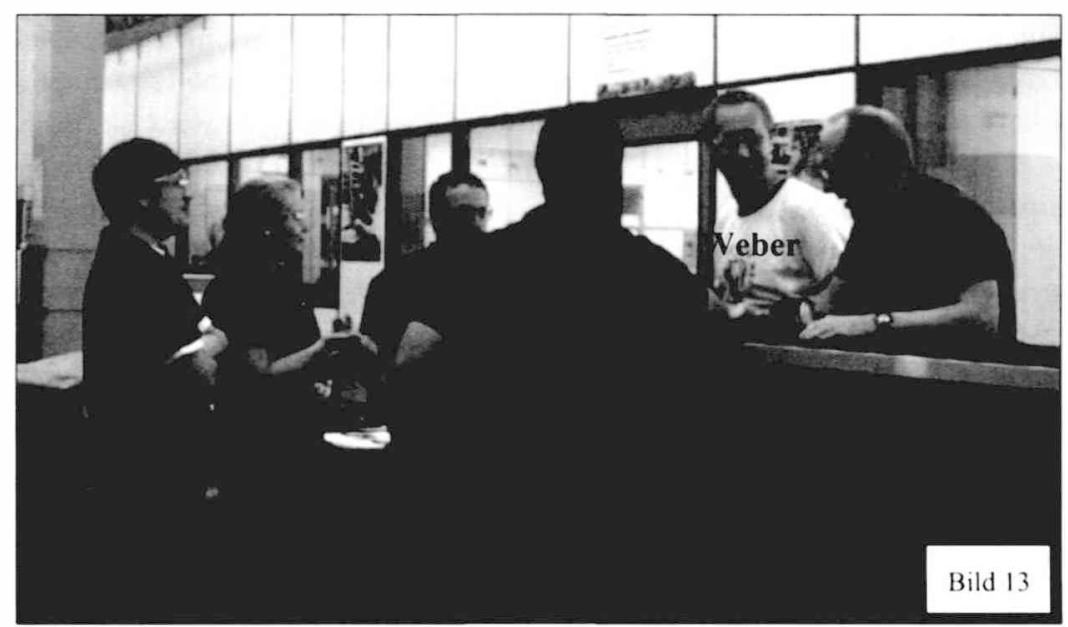

115 Maxi wir Konnten ah ${ }^{\circ}$ hh ähm-

(ilcichzeltig dreht auch Hannes seinen Kupf nach rechts zur Kamera und sicht direkt hincin. Es wird deutlich. dass Weber. Peter und Hannes, sich auf der Girundlage wechselselliger Wahrnehmung koordinieren und dadurch ihre konvergierende OrientIerung auf den zweiten Interaktionsstrang zeigen. Dies geschicht. als Maxi gerade

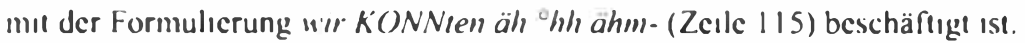

Als Weber dann seinen Kupf emeut nach unten senkt und wie sein Kollege das Aufinahmegerät lixiern, begınnt Hannes seinc Äußcrung ja herr Offenhe'im; hille $L \vec{A}$ (heln: (Zeile 117f.). Bislang uar Verbalıtat als Ressource ausschlicßlıch für dic argumentalive Auscinanderset<ung "reservierte. (hris und Peter hatten <uerst in einer Dyade und später in eıner Trıade zusammen mil Hannes den zweilen Fukus gänzlich ohne den Einsate verbaler Ressourcen whearbeitece. Dadurch wurde die weiterlauliende. primär verbal argumentatıve Auscinandersel<ung nicht gestört. Da nun auch

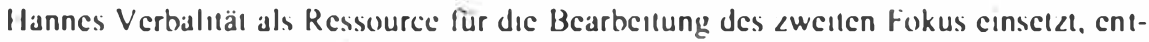
stchı cıne Konkurren $\angle$ mı Maxıs argumentaltiven Bemühungen.

An dieser Stelle \&eigt sich der „Orienticrungs-Switche der (iruppe: Der erstc und der Lweile fokus haben nunmehr ihre Posilion und Relevan getauscht. Hannes hal <u diesem Zeilpunkı berelts erkannt, dass durch die Umorienticrung der Fokusperson $^{h}$ ( Heber) nun der zweile fokus eur primären (iruppenorientierung geworden ist und "traut siche daher. nun auch verbal akıv <u werden.

Auf Hannes verbale Adressicrung von Offenheim reagien Weber dann mit cincm Blıck und cincm Lächeln in dic Kamera. Bei der Reaklion hille L.Acheln:

6 Das Konzept "tiokusperson u uird erstmals skiz.zicrt in Schinılt Deppermann (200?) und in Schmilt, Deppermann (201(0) Heiter ausgefühn (siche auch Schmitt 2010 und 2013). Das Konzept ıst eın Beıspicl für dıc Notucndigkeıt. für dic multımodale Intcrakıonsanalysc gegenstandsadäquate Konzepte zu entu ickeln. dic souohl der audio-visucllen Spezifik der zugrundelicgenden Intcraktıonsdokumentc als auch organısatıonsstrukturellen Relevanzen der analysierten Situation Rechnung tragen. 


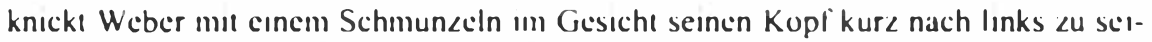
nem Kollegen, worauthın dieser mentgeistert" zur Kamera blıckt.

\section{Maxi}

Zu Beginn des Segments vertrilt Maxı die Interessen des Teams. Sie steht und hal die rechte Hand in dic Hüfte gestemme, während sic sich mil der linken Hand auf dem neben thr stehenden Arbeitstisch stülat. Als Offenheim die Kopthörer zu sich «icht und Chris unter lautlosem Lachen rhythmisch seinen Oberkörper bewegt, beginnt Maxı thre verbalen Auslührungen durch gestıkulatorısche Aktıvitäten zu unterstützen. Sic gestıkulien ab dem Moment, an dem Offenheim die Kopfhörer aufsetat und Weber mit scinem Lächeln berests erstmalıg auf die Anwesenhell seines Kollegen reagiert

Dic einselzende Gestıkulatıon kann als onlıneanalytısche Reaklıon auf dic Präsenzfigur Offenheims gesehen werden. Sein expluratives Verhalten und die ersien interakuven folgen, die sich im Verhalien einzelner Ensemblemitglieder zeigen. nımm Maxı als konkurricrend und störend wahr. Sic reagient auf diese Störung, indem sıe neben Verbalıtät nun auch Giesukulation einset८l. um die Aufmerksamkeıl Webers beı sich zu behalten.

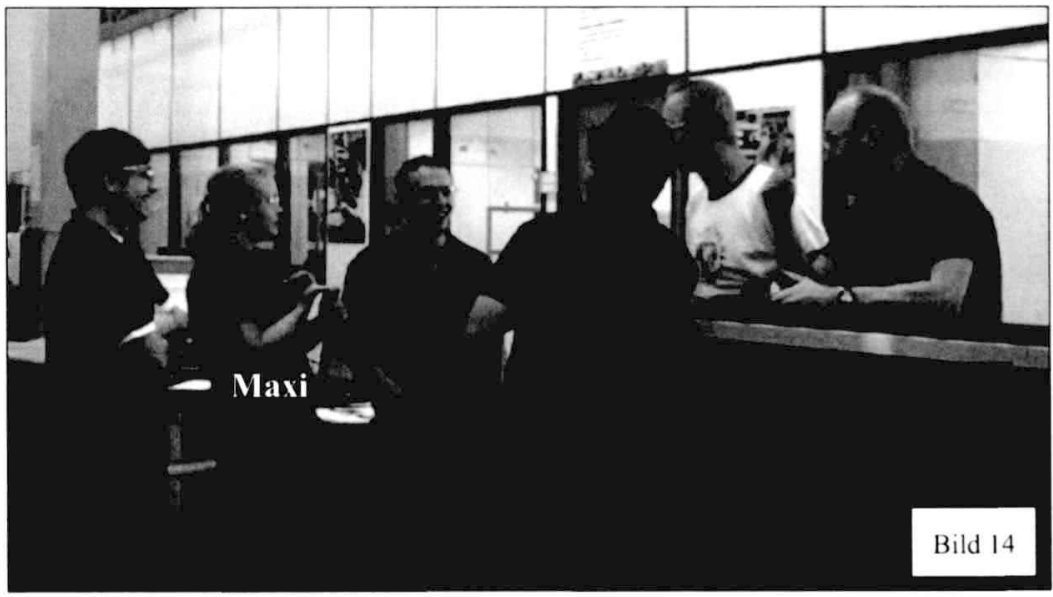

113 Maxi (.) we:l war kcnnter. die die die plare nithe vollerder.

Zu diesem Zeitpunkı lachen ( hris und Hannes. die jewells links und rechts neben thr stehen. bereits lautlos über Offenheim. Als Reaktıon auf die immer stärker wierdende Relevan Offenheims veränden Maxı nun auch ihre Körperposilur. Sie gibı ihre bisherige Verankerung auf, bewegı sich etwas nach vorne. löst dabes thre linke Hand und setzl diese als gestikulatorische Ressource ein. Indem sie mit ihren fingem dic ein 2 inen Argumente aufiahlt. Dabes ist sic blicklich und körperlich auf Weber fokussicre [Bild 14].

Bevor Peter mit Chris erstmalig eine Dyade etablicen. blickt Maxi kurz Ofienheim ins Giesicht. Dic onlıneanalytische Verarbellung der störenden Anwesenhell des zwillen Ausbilders hinterlässt auch in der verbalen Vertrctung der Ciruppe ihre Spuren: Dic Relevanz des aweilen Fokus kopıert sich gewissemaßen in die Äußerungs- 
struktur. wie man an der formulıcrung weil wir kommen die die die PL.Ane nichs vollenden. (Zeile 113) zeigt.

Maxi, die sonst schr Müssig und ohne Wiederholungen formuliert, realısicr ene zwcifache Anikelwiedcrholung die die die PLAne. Dicse crfolgt, als ihr Blick zu Offenheim geht. Während sic also die thematische Entwicklung ihrer Äußerung für eınen Moment aussetzt, indiziert sie mit ihrem Blıck den Verursacher, der durch sein interakıves Verhalten eine konkurtierende Relevanz etablicrt hat und dadurch dic Bearbeıtung des ctablıcrten fokus waufhälı巛. Dies verdeutlichı sıe gewisscrmaßen durch ihre formulierungsweise.

Noch cin weiteres Mal hınterlässı die kunkurricrende Relcvanz Spuren in Maxıs Äußcrungsproduktion. Als sic cin wetteres Argument anführt, Ist ihr Redefluss durch Hesitatıonsphänomene und längeres Ausatmen charakterisicrt: wir KONNıen äh ${ }^{\circ}$ hh ähm- (Zcilc 115). Dicsmal reagien sie aul eıne Kopidrchung des Ausbilders Weber zu seinem Kollegen. In diesem Moment gibe dic Fokusperson ihre bi-fokale Orıenticrung auf und wendet sich der monofokalen Bcarbeitung des zweiten fokus zu. Maxi versucht noch ein letztes Mal. dic argumentatıve Auscinandersctzung aufrecht $<\mathrm{cr}$ halten. Sic formulien ein weiteres Argument, gibe dann jedoch auf und briche ihre in Entwicklung belindliche Äußerung (waren jo alle's PLAne die die, Zcilc 119) ab [Bild |5].

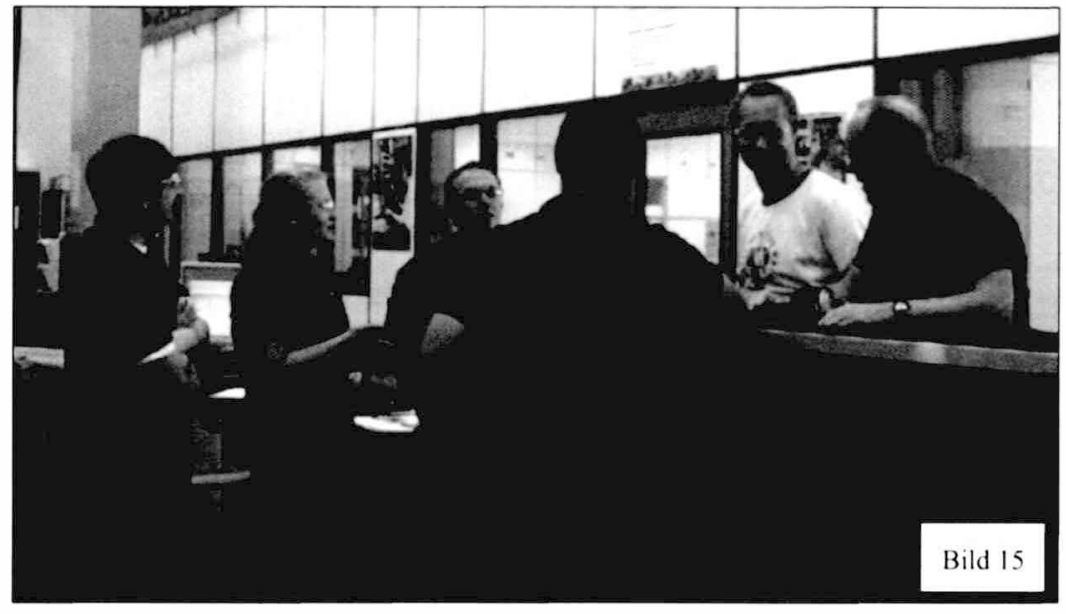

116 Maxi die pneuMAl: ksacter net fertig steller,

Sic reagierı mit diesem Abbruch darauf, dass bereits alle anderen Ensemblemitglicder sich ausschlicßlıch auf den $2 w e$ elen Aushilder orienticrt haben und sic selbst inzwischen in der argumentatıven Auscinandersetzung "verwaıst" Ist 


\section{Im Lachen vereint?}

Mil dem Abbruch von Maxis Äußcrung beginnen ('hris, Peler und Hannes, stımmhaft immer lauter werdend zu lachen. Weber, der sich blicklich seinem Kollegen Oflenheim zugewandt hat, hebt seine lınke Hand mil offener Handlläche zur Kamera und wischt in der Luft hinter dem Kupl seines Kollegen hın und her [Bıld 16].

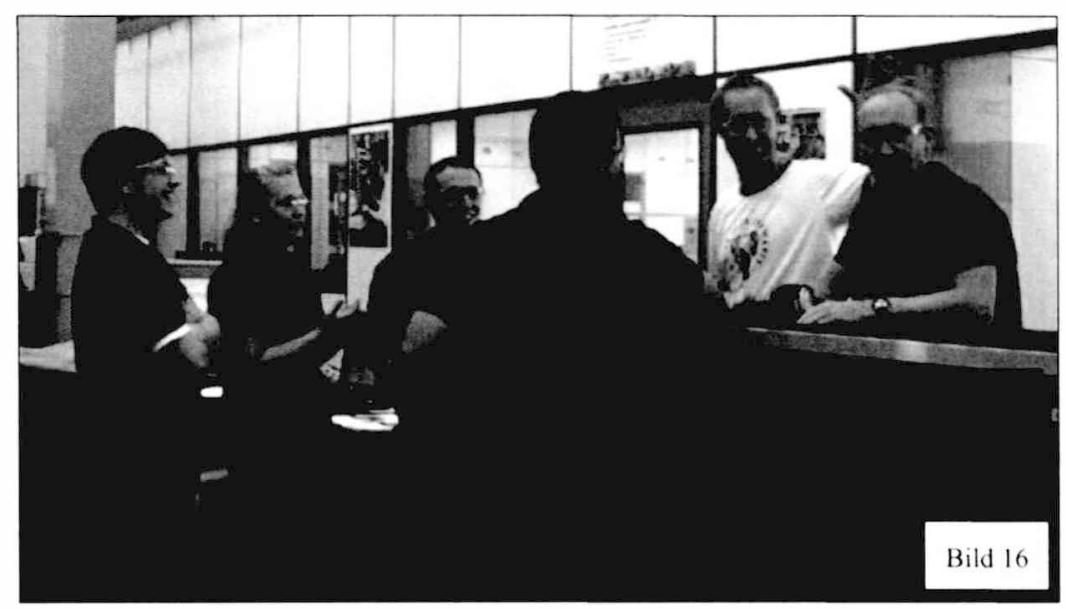

Offenheim löst daraulhın scinc Hände sofort von der Werkbank. hebt sic in die Lufi, als hälte man ihn auf frischer Tat errappe und als wolle er sich "ergebene. Diese (ieslikulation wird begleilet von einem entgeisteren Blick in dic Kamera. Unmittelbar danach verlässt er den Interaktıonsraum des Ensembles und tritt nach links aus dem Bild.

In diesen dynamischen Abgang hınein setzen das Lachen des Ensembles und die verbale Bearbestung ein. Peter reagiert mit him him hille LA Ascheln: (Zeile 122) und zılıcr damı fast wörtlich dic zuvor von Hannes realisierte Äußerung ja herr () flenheim: hille LÄcheln: (Zeilen 1171.). Diese Außcrung selzl erneut ein kullektives Lachen in Gang, an dem sich alle Auszubıldenden beteiligen. Nun zeigt auch Weber beı cinem lautlosen l.achen kurL seine Zühne

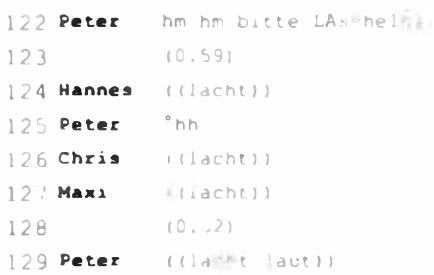

EInc emeute Lachsalve wird dann durch dic Außcrung Webers hat er.s geMERKT: (Zcile 131) motivicri. der damı aul die spätc und dadurch lür das Enscmble unter-

7 Klassische Arbeiten zu "Lachen in der Intcraktiona sind Jefferson (1979. 1987), siche wei心̈rhın, d心 für unseren Zusammenhang interessantén Arbeiten von (ilenn (2003), sou ic als neucre systemalische Zusammenstellungen Wagner Vöge (2010) und llolt (2011). 
haltsame Erkennınıs scines Kollegen verweist Als Reakıon hebı Maxı Ihre Hände ¿um Gesichı und hälı sich den lachenden Mund zu.

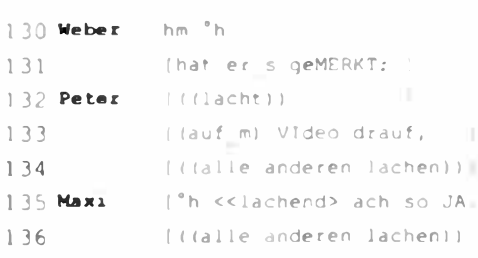

Dic lachende und unterhaltsame Bearbeilung des Abgangs von Offenheim wird durch eıne weilgehende Synchronisierung des Verhaltens der Ensemblemilglieder charakterisicrt. Alle Betciligten schauen zur gleichen Zeil Offenheim hinterher. wobei Peter aufgrund sciner Posilion seinen Oberkörper extrem verdrchl, um sich dem kullckııen Blick anzuschließen [Bild 17].

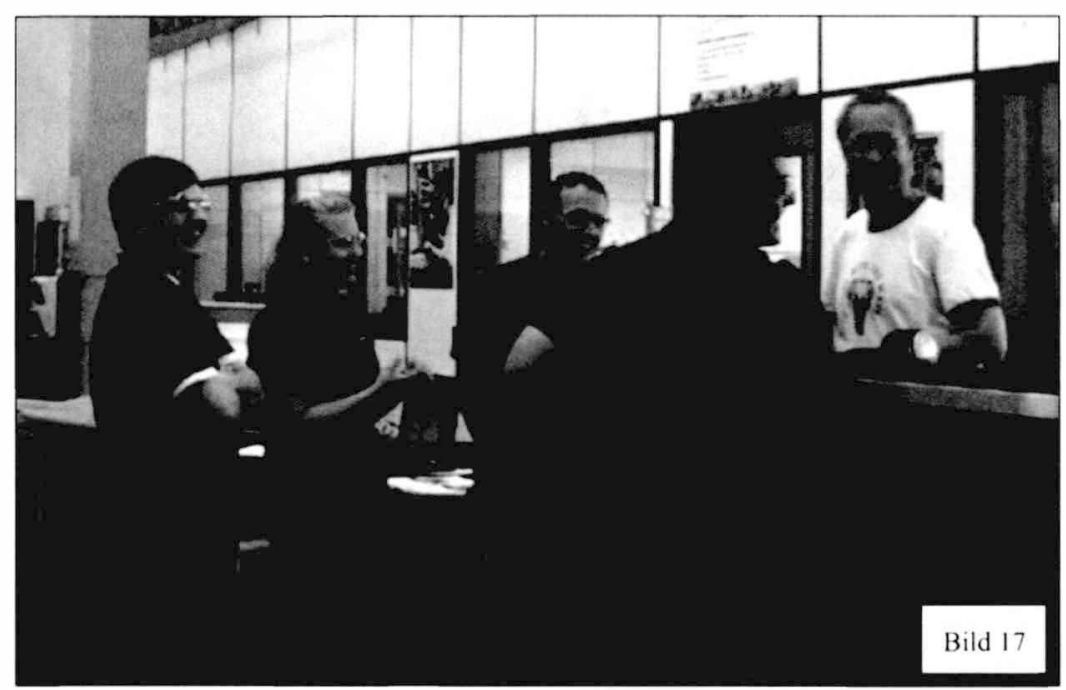

Alle Beiciligten blicken mehrfach, jedoch zeillich verseızl, dirckı in dic Kamera. PeIer ahmi <udem Offenheim nach, Indem er pantommisch darstellt. wie er sich Kopfhörer aufselzı. Schlıeßlich tragen - anders als bei der argumentatıven Auscinander-

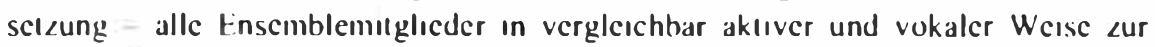
Konstitution dicser unterhaltsamen Phase bet.

Interessanterweıse wiederholt Maxı, dic als L.et<te und etwas verspätel der neuen (iruppenuricnticrung gefolgt war. in dicser Phase all dic Entwicklungsschrittc. welche dic anderen Betciligten bereits $\angle u v o r$ be der schriltweisen Etablicrung des $\angle w e 1-$ ten fokus rcalisien haben: Zunächst lächclt sic laullos, «cigt dann ihre Zähne und begınnı darauthın ımmer lauter werdend $<$ lachen. Schlıeßlıch sicigen sic sich in cinen expressiven Ausdruck hinein. bei dem sic ihren Oberköper nach vorne beugi. mıl beiden Händen ihren Mund bedeckt [B।ld 18] und ihren Körper unter Lachen ins Hohlkreu< bicel [Bıld 19]. 

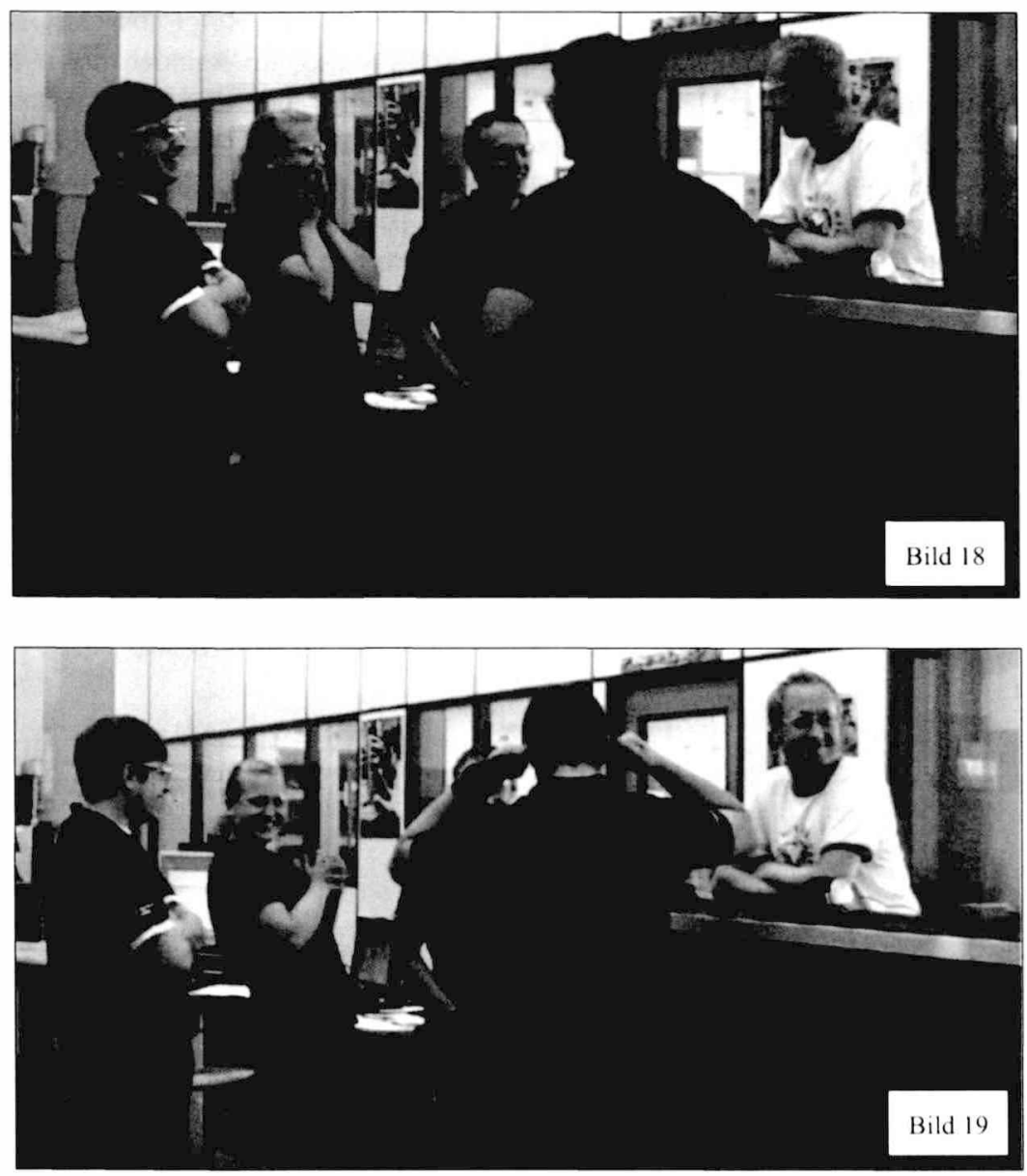

Dicses Verhalten zeigt Maxi jedoch erst nach der Äußcrung des Ausbılders hal er.s geMERKT: (Zcile 131), als dieser sich berests wieder körperlıch und blicklich auf sic orienticr hat. Dies ist die Vorausset<ung dalür, dass das Enscmble nun wieder $\angle u$ Fokus 1 <urückkchren kann.

Maxi, die sich selbst schnell wieder auf den Ausbilder ausrichtet, nutzt die reetablierte dyadische Struktur zwischen Weber und ihr, um den Wiedereinsticg in dic argumentatıve Auscinandersetzung auf den Weg zu bringen. Bei Weber, der sich körperlich wieder auf Maxı ausrichtet, verschwindet dann auch rasch das Lächeln aus seinem Giesscht.

\subsection{Rückkehr zur argumentativen Auseinandersetzung}

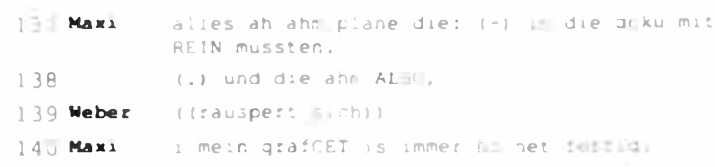


Die Re-Etablicrung der argumentativen Auscinandersetzung erfolgt also durch dic Auszubildende. die zuvor letzte etablierte Sprecherin war, im Rahmen der dyadıschen Struktur, die vor der Unterbrechung bestanden hattc. Dass Maxı auch während der unterhalisamen Unterbrechung ihre Orientierung auf fokus I nicht aufgegeben hat. verdcutlıcht sic. ındem sic mut alles äh ähm pläne die: genau ihre abgcbrochenc Äußerung (waren ja alles PLÄne die die-, Zcılc 119) wicder aufgreift und nunmehr becndel [Bild 20].

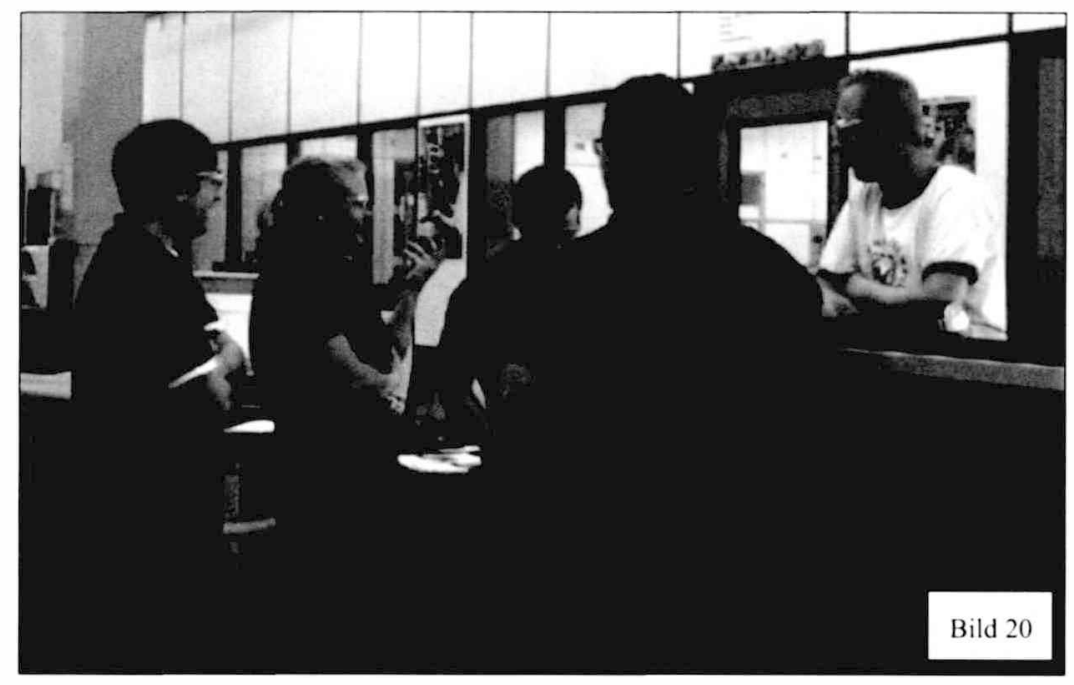

\section{Fallanalytisches Resümee}

Dic schrittweise Herstellung der Unterbrechung geschicht in einem langgestreckien Prozess, an dem sich sukessiv immer mehr Ensemblemitglieder beteiligen. Aus einer <unächst ersien individuellen Relevanz eines Beteiligten entsichen weiterc. gleichsinnige individuclle Relevan 2 en. die sich dann zunächst in ciner Dyade und dann in einer Triade intersubjektıv verfestigen. Als sich mı Weber die Fokusperson des Ensembles umorienlier. Hannes den zuciten fokus verbal intersubjekliviert und schlıcßlıch auch Maxı ihre Orıentierung auf die argumentative Auscinanderset<ung aufgibt. hat sich eine stabile. ncue und kolleklıve Oricnlıcrung herausgebildet. Das Enscmble ısı dann - als Höhepunkı dieser Entwicklung vollständıg im kollckıven Lachen über den zweiten Ausbilder vereınt.

Dıc argumentatıve Auscinanderselzung wird temporär gän/lich aufgegeben, als Weber seinen Kopf nach lınks «u seinem Kollegen wendel und dadurch dic Zwischen ihm und Maxı exıstierende Argumentations-Dyade auflöst. Es glbt also neben dem Slatus des Ausbilders. der es für Maxı schwieng macht. nach dessen Abuendung dic Auscinandersetzung wetter aufrecht zu crhalten. ein interaktionsstrukturelles Argument dafür, sıch ebenfalls der Orıentierung der andem an 2 uschließen. Fast als »Bearbellung" ihrer verspäteten Koordination mil der Orientierung der anderen Ensem- 
blemıtglieder stımmt sie dann expressiv und inszenatorısch in das kolleklıve Lachen über Offenheim cin. Ihre expressive Panizipation am Lachen hat in gewisser Hınsıchı dıc Qualılät ciner Selbsıkurreklur.

Die Relevanz der verbal abstınenten Gruppenmutglieder für dic Elablierung der unterhaltsamen Unterbrechung kann man beispielhali bei (hris schen. der fres ist von verbalen Verpllichtungen und argumentativen Aulgaben. Dadurch hat er genügend Kapazitäl, die Präsenzligur des zweiten Ausbılders in ihrer Angebolsqualitä zu erkennen. durch Monituring abzusichem und mıl seinem eınsel<endes Lachen auch für de anderen Ensemblemighleder kund zu tun.

\section{FUNKTIONALE IMPLIKATIONEN DER UNTERBRECHUNG}

Beı der analysierten Ko-Kunstruktion handelt sich um eine temporäre, unterhaltsame Vergesellschatiung (Simmel 1983) des Ensembles. deren Zweck sich im gemeinsamen Lachen über einen Außenstehenden realısiert. Die Präsen<tigur des \&weilen Ausbilders und seine exploralıven Aklivitiaten be gleich 2 illgem Nichtwissen um weseniliche Siluationssirukluren (Aufnahme mil Video) sind dic eentralen Voraussetzungen für die Herstellung der unterhaltsamen Unterbrechung. Das gemeinsame Lachen stellı dann den Höhepunkı der ko-kunsirukliven Herstellung dar.

Der \&entrale Punkt besicht nicht so schr im gemeinsamen Lachen der Auszubildenden mil ihrem Ausbilder. Es ist vielmehr die lachende Variante, sich gemeinsam über eınen anderen (Außenseller). der nicht zur eigenen Gruppe gehört. lustig zu machen. Der Außenstehende ist für die über ihn Lachenden liunktional. weil er de (iruppenzugehörigkell $\mathrm{m}$ Lachen der Einzelnen themalısch und intersubjeklıv erlahrbar macht. Unter diesen Bedingungen realisieren sich die posiliven Implikaltionen der Herstellung und der lachende Vollzug der Unterbrechung: die unterhaltsame und von deutlicher Intersubjeklıvilat getragene temporäre Aus\&ell, die für die weitere argumentative Auscinanderset<ung eın Moment der Entspannung und Entlastung produ<sert

\section{MULTIMOdALITÄT DER KO-KONSTRUKTION}

Sich ko-konstrukliv zu engagieren scheint unter bestımmten situativen und interaktionsstrukturellen Bedingungen einen hohen sozialen Wer $\angle$ haben. Spezilische Bedingungen können jedoch auch dazu lühren. dass es Kosten hat, sich nicht kokunstruklıv zu engagieren. Dann ist das ko-konstruktıve Engagement niche mehr so schr eıne molivierte Seleklion aus einer Viclzahl liunklional und sozial äquivalenter Alternativen, sondern die erwartbare und erwantete Verhaliensweise.

In unserem fall trifli das lür Maxı zu. Sıe gerät als tetzle Verreterın der (iruppenınteressen in eine Siluation. in der sie die Umorientierung aller anderen Ensemblemitglieder "verpasst " hat. Sic bearbellet ihren verspäteten Eintrill in dic neuc (iruppenorienticrung durch eine besonders deulliche und exalticrle Parlizipation am kollekuven Lachen über den zweilen Ausbilder. 
Würde sie nicht akıı am "sich über den Unwissenden lustig machen «cilnchmen. stünde sic - obwohl sie die Ietzte aufrechte Venteterin der Gruppeninteressen Ist - sıtuatıv außcrhalb der lustıgen Vergescllschafiung. Sic wüde sich dadurch interakıonsdynamısch und hınsichilıch der Modalität der Vergesellschaftung isolicren. Ihr ko-konstruktıver Beıtrag ist dic Voraussctzung dafür, dass sich das Ensemble als Ganzes sıltuatıv im Lachen über eınen Außenstehenden solidarisieren kann.

Dic multimodale Perspeklive und die aufeinander bezogenen Konzepte "Interaktoonsraum" und "Interaktionsensemble" machen deutlich. was an analytischer Einsicht alles verloren geht. $u$ enn man Interaktion allgemeın und ko-kunstruktıve Strukturen/Aktivitäten im Besonderen primär oder ausschließlich unter dem Aspekt der Verbalıäı fokussicrı

Dies gile bercits für die Gruppenstruklur der Auszubildenden und deren interne Differenzierung in dicjenigen, d心 d心 argumentative Auscinandersetzung mit dem Ausbilder Weber für de (iruppe führen (Maxi und Hannes), und den verbal Abstınenten (Chris und Peter). die dic Gruppe und deren Relevanzen durch koordiniente Präsenz im gemeınsamen Interaklionsraum verkörpern. Die beiden Letzıgenannten ¿u überschen. was bei ciner ausschließlich verbalen Analyse - ¿umindest in der Phase der ausschließlichen Relevanz von fokus 1 - zwangsläufig geschehen würde. würde deren grundlegende runktıonalıtät und damit eın wesentliches Strukturelement der gesamten Siluation ignorieren. Ihr Verhalten muss viclmehr unter der Perspektıve qualıtativer Interaktionsbetciligung im Rahmen einer grundlegenden Arbeitsteilung betrachtet werden. Unter dieser Perspektive besitat bereits dic interaktionsräumlıche Repräsentanz der (iruppe ko-konstruktıve Qualität.

Offensichtlich wird die ko-konstruktıve Wichtigkeıt der beiden verbal abstınenten Gruppenmitglieder in ihrer Funktionalität beı der Herstellung der unterhaltsamen Unterbrechung. Sic sind diejenigen. die den zweiten fokus als solchen erkennen und inn den andern tnsemblemigliedern anbieten. Sic repräscnticren die ersic intersubjekliv abgesichertc Relevan neuen fokus bezogenen Dyade. Dies sichibar zu machen, ist eine wesentliche Leislung der multimodalen Perspeklive.

Hier zeigt sich in eindringlicher Weise dic Relevan der von den Herausgebern in ihrem Beitrag formulierien Forderung. dass "jedes kummunikallive Ereignis Beschreibungen fordert, in denen der Beitrag aller verfügbaren Handlungsressourcen zur Sinnkonstitution und ihr Zusammenspiel im interaktıven Geschehen zum Gegenstand der Untersuchungen gemache werden “ (Selte 35) muss.

Interessant ist schließlich. dass sich in der ko-konstruktiven Herstellung der Unterbrechung dic hicrarchischen Strukturen der Ausbildungssiluation reproduzicren. Erst als der Ausbilder Weber seien Kollegen für das Amüsement des Ensembles freigibi, secu das kollektıve Lachen eın.

\section{LITERATUR}

Deppermann. Amulf Schmılt. Reınhold (2007): "Koordination. Zur Begrundung cines neuen forschungsgegenstandes", in: Reinhold Schmilt (lig.): Koordination. Analysen zur multumodalen Intcraktion. Tübingen: Narr. S. 15-54. 
Diaz, Felıx/Antakı, Charles/Collıns, Alan F. (1996): "Usıng completion to furmulate a statement collectuvelyu, in: Journal of Pragmatics 26, S. 525-542.

Cilenn. Phillip J. (2003): Laughter in intcraction. Cambridge: Cambridge Universily Press.

(joodwin. Marjoric H. (1983): "Searching for a word as an interactive aclivily", in: John N. Decly/Margor D. Lenhan (Hg.): Semolics 1981. New York: Plenum Press. S. 129-138.

Goodwin, Marjoric H./Goodwin. Charles (1986): "Gisture and coparticipation in the acuvity of scarchıng for a wordu. in: Thomas A. Sebeok (Hg.): Semiotica. Volumce $62(1 / 2)$. S. $51-75$.

Hausendort. Heiko (2003): "Deixis and speech siluation revisiled. The mechanism of perceived perceptione, in. Fricedrich l.enz ( $\mathrm{Hg}$.): Deiclic conceptualisation of space, lime and person, Amsterdam/Philadelphia: John Benjamıns, S. 249-269.

Hausendort. Heiko/Mondada, Loren<a/Schmill, Reinhold (2012): "Raum als interaklive Ressource: Einc Explikation «, in: Dies. (HIg): Raum als interaklive Ressource. Tübingen: Narr. S. 7-36

Heidtmann, Danıcla/töh. Marıc-Juan (2007): "Verbale Abstınene als form interaklıver Betciligung", in: Reinhold Schmilt (Hg.): Koordination. Analysen <ur multimodalen Interaktion. Tübıngen: Narr, S. 263-292

Holt, Elisabeth (2011). "On the nature of "laughables": Laughter as a responsc to overdone liguratıve phrasese. In: Pragmatics 21/3. S. 393-410.

Jefierson, Gall (1979): "A technique for inviling laughter and tis subsequent acceptanceddeclination «. in: (icorge Psathas ( $\mathrm{Hg}$.): Everyday language: studies in ethnomethodulogy. New York: Irvington. S. 79-96

Jeflerson. Gaıl/Sacks. Harvey/Scheglofi, Emanucl A. (1987): "Notes on laughter in the pursuit of intimacyu, in: (iraham Button/Juhn Lece (Hg.): Talk and social organization. Clevedon: Multilingual Matters. S. 152-205.

Lemer, G. H. (2002): "Tum-sharıng: the choral co-production of talk-in-interaction In: C. Ford/B3. Fox/S. Thompson ( $\mathrm{Hg}$.): The Language of Turn and Sequence. Oxford. Oxford Universily Press, S. 225-256

Lerner, G. II. (2004): "Collaborative Turn Sequencese, in: Ders. (Hg.): Conversalion Analysis. Studies from the First (iencration, Amsterdam: John Benjamins, $S$. 225-256

Mondada. Loren 2 a (2007): "Interaktionsraum und Koordinierung". in. Reinhold Schmılt (Hg.): Koordination. Analysen zur multumodalen Interaktion, Tübıngen Narr, S. 55-93.

Muller, Cornclia/Buhle, Ulrike (2007): „Das Fundament lokussiener Interaktion. Zur Vorbercilung und Herstellung von Intcraktıonsräumen durch körperlıche Kourdınation «, in. Reinhold Schmılı ( $\mathrm{Hg}_{\text {g }}$ ): Kourdination. Analysen zur multimodalen Intcraklion. Tübıngen: Narr. S. 129-165.

Sacks, Harvey (1992): "(illaboratives; possible ullerances; ullerance pairs; greelings and introductions«, in: Lectures un conversattion. Vol. II. Lecture 5. S. 56-66.

Schmitt. Reinhold (1992a): Dic Schwellensicher. Sprachliche Präsen und sozialer Austausch in cincm Kıosk, Tübıngen: Narr.

Schmıtt, Reinhold (1992b): "Das Konzept der Präsenaligur. Ein Beltrag zur Integration von Konversationsanalyse und objekliver Hermeneutik «, in: Protosoziologic 3. S. 123-131 
Schmılt. Reınhold (1998): "Unterstützen Im (iespräch. Zur Analyse manıfester Kooperationsverlahren«. in: Zeitschrift für Sprachwissenschaft 16/1-2 (1997). S. 5282.

Schmıtt, Reinhold (2010): "Verlahren der Verstehensdokumentation am filmset: Antizipatorische Initlativen und probeweisc Konzeptrealisicrung«. In: Amulf Deppermann/Ulicich Reitemeicr/Reinhold Schmit/ThomasSpranz-Fogasy ( $\mathrm{Hg}$ ): Versichen in professionellen Handlungsfeldern. Tübıngen: Narr. S. 210-362

Schmıtt. Reınhold (2012 a): "(ichen als situierte Praktık: ,Gemeinsam gehen" und .hınter jemandem herlaulen" ". in: Gesprächsforschung Onlıne. Ausgabe 13, S. 1 44 (www.gespracchsforschung-ozs.de).

Schmıll, Reinhold (2012 b): "Körperlıch-räumlıche (irundlagen interaklıver Betcilıgung: Das Konzept .Interaktionsensimble" ", in: Heıko Hausendort/Loren La Mondada/Reınhold Schmılt. Reınhold (Hg.): Raum als interakıve Ressource. Tübıngen: Narr. S. 37-87.

Schmııt, Reınhold (2013): Körperlıch-räumliche Aspekte der Interaktıon, Tübıngen: Narr.

Schmılt. Reinhold/Deppermann. Arnulf (2007): "Monitoring und Koordination als Vorausset<ungen der multımodalen Konstitution von Interaklionsräumen“, in Reinhold Schmitt ( $\mathrm{Hg}_{\text {. }}$ ): Koordination. Analysen zur multımodalen Interakiıon. Tübıngen: Narr, S. 95-128.

Schmılt. Reinhold/Deppermann. Arnulf (2010): "Dic Transition von Interaktionsräumen als Situationseröffnung", In: Lorenza Mondada/Rcinhold Schmıtl $\left(\mathrm{Hg}_{\mathrm{g}}\right)$ Situationseröfinungen: Zur multımodalen Herstellung fokussiener Interaktion. Tübıngen: Narr. S. 335-386.

Simmel. Alfred (1983): Sozıologic. Untersuchungen über die formen der Vergesellschaftung. Berlin: Duncker \& Humbolt

Wagner. Juhannes/Vögc. Monıka (Hg.) (2010): "Laughter in interactionu. In: Special issuc in honor of (jall Jefferson. Journal of Pragmatics 42/6, S. 1469-1576. 